\title{
Lagrangian theory of gravitational instability of Friedman-Lemaître cosmologies - second-order approach: an improved model for non-linear clustering
}

\author{
Thomas Buchert and Jürgen Ehlers \\ Max-Planck-Institut für Astrophysik, Karl-Schwarzschild-Str. 1, D-85740 Garching, Munich, Germany
}

Accepted 1993 March 12. Received 1993 March 8; in original form 1992 July 23

\begin{abstract}
A BST RACT
A large class of solutions for second-order irrotational perturbations is derived in the framework of the Lagrangian theory of gravitational instability of a homogeneous and isotropic universe investigated in earlier papers. The solutions are evaluated in detail for perturbations in a flat background universe. The form of the solutions is designed for use in studies of the formation of large-scale structure from generic initial conditions. Some general remarks on the properties of the solutions are made. The result is illustrated by a special case and discussed. In particular, it is found that sheetlike structures stay compact after shell-crossing (as in the competing 'adhesion model'), and that the collapse of first objects occurs earlier (as expected from numerical simulations) in the second-order approach. Both these properties compensate shortcomings of the 'Zel'dovich approximation'. In contrast to the 'adhesion model', the $n$ th-order Lagrangian perturbation solutions also describe internal structures of self-gravitating pancakes $(=2 n+1$ stream systems) in terms of the $n$th orbit crossings within pancakes.
\end{abstract}

Key words: instabilities - methods: analytical - galaxies: clustering - cosmology: theory - large-scale structure of Universe.

\section{OVERVIEW}

In a recent paper (Buchert 1992, henceforth B92), the Lagrangian theory of gravitational instability of FriedmanLemaitre cosmologies was investigated and solved up to the first order of the deviations from homogeneity. It was shown that the 'Zel'dovich approximation' (Zel'dovich 1970, 1973) can be considered as a subclass of first-order irrotational perturbation solutions in this theory. These solutions were first given in an earlier paper (Buchert 1989), in which it was demonstrated that the first-order solutions provide exact solutions in the case of plane-symmetric inhomogeneities as well as in a special three-dimensional case, where an invariant definition of the solution class is the vanishing of two eigenvalues of the peculiar-velocity gradient.

In the present paper, we push this theory to the second order. We restrict ourselves to irrotational perturbations. We derive a large class of second-order solutions, evaluate it in the case of a flat Friedman background, and express the result in terms of the initial conditions for the peculiar velocity and peculiar acceleration. This approximation is then discussed and illustrated in a special two-dimensional case and compared to the first-order approximation.

We obtain a description that is applicable to studies of the formation of large-scale structure from generic initial conditions. Thus the present work provides an improved approximation for non-linear gravitational instability, which takes into account important aspects of the tidal action of selfgravitating 'dust' continua in the non-linear regime. In particular, this approximation compensates shortcomings of Zel'dovich's approach concerning, e.g., the formation of first objects and the compactness of sheet-like structures after shell-crossing.

In the Eulerian framework, the second-order perturbation theory has been solved and discussed by, for example, Peebles (1980, section II.18 and references therein) and Grinstein \& Wise (1987). Other related articles concern Lagrangian perturbations of a stationary perfect fluid (Lynden-Bell \& Ostriker 1967; Friedman \& Schutz 1978 and references therein). A parallel attempt to set up the Lagrangian perturbation theory in Friedman-Lemaitre backgrounds was pursued by Moutarde et al. (1991), and 
included a comparison of a third-order example with a numerical simulation. Recently, the latter work has been extended to second-order perturbation solutions for non-flat background models by Bouchet et al. (1992), who also discussed applications.

\section{THE EULER-POISSON SYSTEM IN LAGRANGIAN FORM FOR GENERAL AND IRROTATIONAL FLOWS}

In the Lagrangian description, integral curves $\boldsymbol{x}=\boldsymbol{f}(\boldsymbol{X}, t)$ of the velocity field $\boldsymbol{v}(\boldsymbol{x}, t)$ are introduced:

$\frac{\mathrm{d}}{\mathrm{d} t}=\boldsymbol{v}(\boldsymbol{f}, t), \quad \boldsymbol{f}\left(\boldsymbol{X}, t_{\mathrm{o}}\right)=: \boldsymbol{X}$

These curves are labelled by the Lagrangian coordinates $X_{i}$. We can express all fields, such as the Eulerian coordinates $\boldsymbol{x}$ (which are now construed as an additional vector field in Lagrangian space), the velocity $\boldsymbol{v}$, the gravitational field strength $g$, and the density $\rho$, in terms of the field of trajectories $f$ as follows:

$\boldsymbol{x}=\boldsymbol{f}(\boldsymbol{X}, t)$

$\boldsymbol{v}=\dot{\boldsymbol{f}}(\boldsymbol{X}, t)$

$\boldsymbol{g}=\ddot{\boldsymbol{f}}(\boldsymbol{X}, t)$

$\rho=\stackrel{\rho}{ }(\boldsymbol{X}) J^{-1}, \quad J:=\operatorname{det}\left[f_{i, k}(\boldsymbol{X}, t)\right]$.

The comma in the subscript denotes partial differentiation with respect to the Lagrangian coordinates, the dot denotes the Lagrangian time derivative $\mathrm{d} / \mathrm{d} t:=\left.\partial_{t}\right|_{x}+v \cdot \nabla_{x}=\left.\partial_{t}\right|_{X}$; the comma and the dot commute.

Recall that mass conservation is guaranteed by $(2 \mathrm{~d})$ irrespective of any equations that the trajectories $f$ might obey. The Euler-Poisson system can be cast into a set of four evolution equations for the single dynamical variable $f$ (compare B92 for all details, especially for the equivalence of the Lagrangian and the Eulerian forms of the equations):

$\epsilon_{p q[j} \frac{\partial\left(\ddot{f}_{i}, f_{p}, f_{q}\right)}{\partial\left(X_{1}, X_{2}, X_{3}\right)}=0, \quad i \neq j$

$\sum_{a, b, c} \frac{1}{2} \epsilon_{a b c} \frac{\partial\left(\ddot{f}_{a}, f_{b}, f_{c}\right)}{\partial\left(X_{1}, X_{2}, X_{3}\right)}-\Lambda J=-4 \pi G \stackrel{\circ}{ }(\boldsymbol{X}) ; \quad \stackrel{\rho}{ }(\boldsymbol{X})>0$

(indices run from 1 to 3 , if not otherwise explicitly stated; henceforth, $\nabla_{\mathrm{o}}$ denotes the nabla operator with respect to the Lagrangian frame that commutes with the dot).

One class of solutions of the Euler-Poisson system (3) is formed by the homogeneous and isotropic Friedman-Lemaître models:

$f_{\mathrm{H}}(\boldsymbol{X}, t)=a(t) \boldsymbol{X}$.

Inserting $f_{\mathrm{H}}$ into equations $(3)$, we obtain for the function $a(t)$ the single equation

$3 \ddot{a} a^{2}-a^{3} \Lambda=-4 \pi G \stackrel{\circ}{\mathrm{H}}_{\mathrm{H}}, \quad \stackrel{\circ}{\mathrm{H}}=$ constant $>0$,

the first integral of which is given by Friedman's differential equation $\frac{\dot{a}^{2}+\text { constant }}{a^{2}}=\frac{8 \pi G \rho_{\mathrm{H}}+\Lambda}{3}$,

where $\rho_{\mathrm{H}}=\rho_{\mathrm{H}} a^{-3}$ is the background density.

For irrotational flows we can prove the following Lemma.

In the Lagrangian picture, the vorticity, or the angular velocity $\omega:=(1 / 2) \nabla_{x} \times v$, has to be written in terms of the flow field $\boldsymbol{f}(\boldsymbol{X}, t)$ as follows:

$\omega_{i j}(\boldsymbol{X}, t)=\frac{1}{2} \epsilon_{p q[j} \frac{\partial\left(\dot{f}_{i]}, f_{p}, f_{q}\right)}{\partial\left(X_{1}, X_{2}, X_{3}\right)} J^{-1}, \quad i \neq j$.

$(5 \mathrm{a}, \mathrm{b}, \mathrm{c})$

(Note: the components of $\omega$ can be expressed in terms of the antisymmetric tensor components $\omega_{i j}$ used here as $\omega_{i}=$ $(1 / 2) \epsilon_{i j k} \omega_{j k}$.)

Lemma. For irrotational flows the Euler-Poisson system (3a, b, c, d, e,f) can be replaced by equations $(3 d, e, f)$ and

$\omega_{i j}=0$.

$(5 \mathrm{~d}, \mathrm{e}, \mathrm{f})$

In order to prove this Lemma we have to verify the sufficient implication $\omega_{i j}(\boldsymbol{X}, t)=0 \Rightarrow(3 \mathrm{a}, \mathrm{b}, \mathrm{c})$. In the Eulerian picture, equations $(5 \mathrm{~d}, \mathrm{e}, \mathrm{f})$ imply the existence of a potential $S(\boldsymbol{x}, t)$, $v=\nabla_{x} S$. Using equations $(2 \mathrm{~b}, \mathrm{c})$, we have

$\boldsymbol{g}=\nabla_{x}\left[\left.\frac{\partial}{\partial t}\right|_{x} S+\frac{1}{2}\left(\nabla_{x} S\right)^{2}\right]$

This equation implies $\nabla_{x} \times \boldsymbol{g}=\boldsymbol{0}$, which is equivalent to $(3 \mathrm{a}, \mathrm{b}, \mathrm{c})$.

Q.e.d.

Note that this implication holds for any trajectory, since it is based on equations (2) only. It expresses a purely kinematical property of the flow.

We now derive a large class of second-order solutions of the Lagrangian perturbation theory at the background solutions (4). The reader who is interested in applications of the solutions only may move directly to Section 5 .

\section{THE LAGRANGIAN THEORY OF GRAVITATIONAL INSTABILITY: SECOND-ORDER SOLUTIONS FOR IRROTATIONAL PERTURBATIONS}

3.1 Second-order perturbation approach at a FriedmanLemaître background

Henceforth, we restrict all considerations to the case in which the velocity field has a potential. We consider $f$ to be a superposition of a homogeneous isotropic deformation and a vector function $\boldsymbol{p}$ for the inhomogeneous deformation of the medium as follows:

$\boldsymbol{f}(\boldsymbol{X}, t)=a(t) \boldsymbol{X}+\boldsymbol{p}(\boldsymbol{X}, t), \quad \boldsymbol{p}(\boldsymbol{X}, t)=\varepsilon \boldsymbol{p}^{(1)}(\boldsymbol{X}, t)+\varepsilon^{2} \boldsymbol{p}^{(2)}(\boldsymbol{X}, t)$,

$a\left(t_{\mathrm{o}}\right):=1, \quad \boldsymbol{p}^{(1)}\left(\boldsymbol{X}, t_{\mathrm{o}}\right):=\mathbf{0}, \quad \boldsymbol{p}^{(2)}\left(\boldsymbol{X}, t_{\mathrm{o}}\right):=\mathbf{0}$,

where $\varepsilon$ is assumed to be a small parameter. Note that the first-order solutions provide exact solutions if certain constraints on the initial conditions are fulfilled (see Buchert 1989), and that they agree well with numerical $N$-body simulations of the full system until shortly after the first shellcrossings.

To derive the second-order solutions, we proceed as follows. We consider the source equation (3d) only and solve 
this equation for longitudinal perturbations, i.e. $\boldsymbol{p}=\nabla_{\mathrm{o}} \psi$. We then insert this solution into the remaining evolution equations ( $5 \mathrm{~d}, \mathrm{e}, \mathrm{f})$ to determine the constraints (cf. the Lemma).

Inserting the ansatz (6) into equation (3d) and sorting terms that are linear and quadratic in $\varepsilon$, we obtain two partial differential equations involving expressions that are linear, $\mathscr{L}\left[\boldsymbol{p}^{(1)}\right]$ and $\mathscr{L}\left[\boldsymbol{p}^{(2)}\right]$, and quadratic, $\mathscr{Q}\left[\boldsymbol{p}^{(1)}\right]$, in the perturbations [compare the appendix in B92; $I\left(p^{(i=1,2)}\right)$ and $I I\left(p^{(1)}\right)$, $I I\left(\dot{p}^{(1)}\right)$ denote the first and second scalar invariants of the tensor gradients of $\boldsymbol{p}^{(i=1,2)}$ and $\dot{\boldsymbol{p}}^{(1)}$, respectively; the initial density perturbation is split according to $\left.\stackrel{\rho}{=} \stackrel{\circ}{\mathrm{H}}_{\mathrm{H}}+\delta \dot{\rho}\right]$ :

$$
\begin{aligned}
& \left(3 \ddot{a} a^{2}-a^{3} \Lambda\right)+\varepsilon \mathscr{L}\left[\boldsymbol{p}^{(1)}\right]+\varepsilon^{2}\left\{\mathscr{L}\left[\boldsymbol{p}^{(2)}\right]+\mathscr{Q}\left[\boldsymbol{p}^{(1)}\right]\right\} \\
& \quad=-4 \pi G \stackrel{\rho}{ }(\boldsymbol{X})=:-4 \pi G\left[\stackrel{\circ}{\mathrm{H}}+\varepsilon \delta \dot{\rho}^{(1)}+\varepsilon^{2} \delta \dot{\rho}^{(2)}\right],
\end{aligned}
$$

with

$$
\begin{aligned}
\mathscr{L}\left[\boldsymbol{p}^{(i)}\right]: & =\left(2 \ddot{a} a-a^{2} \Lambda\right) I\left[\boldsymbol{p}^{(i)}\right]+a^{2} \ddot{I}\left[\boldsymbol{p}^{(i)}\right], \quad i=1,2 ; \\
\mathcal{Q}\left[\boldsymbol{p}^{(1)}\right]: & =(\ddot{a}-a \Lambda) I I\left[\boldsymbol{p}^{(1)}\right]+a \sum_{a, b, c} \epsilon_{a b c} \frac{\partial\left[\ddot{p}_{a}^{(1)}, p_{b}^{(1)}, X_{c}\right]}{\partial\left(X_{1}, X_{2}, X_{3}\right)} \\
& =(\ddot{a}-a \Lambda) I I\left[\boldsymbol{p}^{(1)}\right]+a\left\{\dot{I} \dot{I}\left[\boldsymbol{p}^{(1)}\right]-2 I I\left[\dot{\boldsymbol{p}}^{(1)}\right]\right\} .
\end{aligned}
$$

The homogeneous deformation (4) solves these equations for $\boldsymbol{p}^{(i=1,2)}=\boldsymbol{0}$.

We now consider any longitudinal part $\{\boldsymbol{p}\}$ of the perturbations only (denoted by the curly brackets around $\boldsymbol{p}$ ). For technical reasons we introduce the vector fields $\{\boldsymbol{A}\},\{\boldsymbol{A}, \boldsymbol{A}\}$ and $\{\boldsymbol{B}, \boldsymbol{C}\}$, which have the following properties. For given arbitrary vector fields $\boldsymbol{A}, \boldsymbol{B}$ and $\boldsymbol{C}$, let $\mathscr{T}(\boldsymbol{A}), \mathscr{T}(\boldsymbol{A}, \boldsymbol{A})$ and $\mathscr{T}(\boldsymbol{B}, \boldsymbol{C})$ denote scalar functions such that

$$
\begin{array}{r}
\Delta_{\mathrm{o}} \mathscr{T}(\boldsymbol{A})=I(\boldsymbol{A}), \quad \Delta_{\mathrm{o}} \mathscr{T}(\boldsymbol{A}, \boldsymbol{A})=2 \cdot I I(\boldsymbol{A}, \boldsymbol{A}), \\
\Delta_{\mathrm{o}} \mathscr{T}(\boldsymbol{B}, \boldsymbol{C})=\sum_{a, b, c} \epsilon_{a b c} \frac{\partial\left(B_{a}, C_{b}, X_{c}\right)}{\partial\left(X_{1}, X_{2}, X_{3}\right)} .
\end{array}
$$

According to a theorem by Brelot, such scalars are guaranteed always to exist; see Friedman (1963). They are not, however, uniquely defined. The gradients of these scalars then provide a longitudinal part of the given vector fields (note that the field $\{\boldsymbol{A}\}-\boldsymbol{A}$, for example, is transverse according to our construction):

$$
\begin{aligned}
& \{\boldsymbol{A}\}:=\nabla_{\mathrm{o}} \mathscr{T}(\boldsymbol{A}), \quad \nabla_{\mathrm{o}} \cdot\{\boldsymbol{A}\}=I(\boldsymbol{A})=\sum_{i} A_{i, i}, \quad \nabla_{\mathrm{o}} \times\{\boldsymbol{A}\}=\boldsymbol{0} ; \\
& \{\boldsymbol{A}, \boldsymbol{A}\}:=\nabla_{\mathrm{o}} \mathscr{T}(\boldsymbol{A}, \boldsymbol{A}), \quad \nabla_{\mathrm{o}} \cdot\{\boldsymbol{A}, \boldsymbol{A}\}=2 I I(\boldsymbol{A}), \\
& I I(\boldsymbol{A})=\frac{1}{2}\left[\left(\sum_{i} A_{i, i}\right)^{2}-\sum_{i j}\left(A_{i, j} A_{j, i}\right)\right], \quad \nabla_{\mathrm{o}} \times\{\boldsymbol{A}, \boldsymbol{A}\}=\boldsymbol{0} ;
\end{aligned}
$$

$$
\begin{aligned}
\{\boldsymbol{B}, \boldsymbol{C}\} & :=\nabla_{\mathrm{o}} \mathscr{T}(\boldsymbol{B}, \boldsymbol{C}), \\
\nabla_{\mathrm{o}} \cdot\{\boldsymbol{B}, \boldsymbol{C}\} & =\sum_{a, b, c} \epsilon_{a b c} \frac{\partial\left(B_{a}, C_{b}, X_{c}\right)}{\partial\left(X_{1}, X_{2}, X_{3}\right)}, \quad \nabla_{\mathrm{o}} \times\{\boldsymbol{B}, \boldsymbol{C}\}=\boldsymbol{0} .
\end{aligned}
$$

Note that the linear or the quadratic expressions enclosed in curly brackets are distributive and commutative in the sense that we can make use of the following assignments: $\{\boldsymbol{A}+\boldsymbol{B}, \boldsymbol{C}\} \leftrightarrow\{\boldsymbol{A}, \boldsymbol{C}\}+\{\boldsymbol{B}, \boldsymbol{C}\},\{\boldsymbol{A}, \boldsymbol{B}\} \leftrightarrow\{\boldsymbol{B}, \boldsymbol{A}\}$. These assign- ments are not, however, bijective mappings, since the potentials $\mathscr{T}$ are not uniquely defined. Likewise, we can write

$\{\boldsymbol{A}\}=\nabla_{\mathrm{o}} \mathscr{T}(\boldsymbol{A})=\nabla_{\mathrm{o}} \Delta_{\mathrm{o}}^{-1} I(\boldsymbol{A})$,

$\{\boldsymbol{A}, \boldsymbol{A}\}=\nabla_{\mathrm{o}} \mathscr{T}(\boldsymbol{A}, \boldsymbol{A})=\nabla_{\mathrm{o}} \Delta_{\mathrm{o}}^{-1} 2 I I(\boldsymbol{A}, \boldsymbol{A})$,

$\{\boldsymbol{B}, \boldsymbol{C}\}=\nabla_{\mathrm{o}} \mathscr{T}(\boldsymbol{B}, \boldsymbol{C})=\nabla_{\mathrm{o}} \Delta_{\mathrm{o}}^{-1} \sum_{a, b, c} \epsilon_{a b c} \frac{\partial\left(B_{a}, C_{b}, X_{c}\right)}{\partial\left(X_{1}, X_{2}, X_{3}\right)}$

(cf. Grinstein \& Wise 1989).

Using (6), (7) and (9), the remaining equations to be solved can now be written as families of ordinary differential equations (labelled by $\boldsymbol{X}$ ) with constant coefficients along each trajectory. We express the first- and second-order parts of the source terms $\delta \rho^{(i=1,2)}$ through the divergence of the initial field strength perturbations $\ddot{\boldsymbol{p}}^{(i=1,2)}\left(t_{\mathrm{o}}\right)$ and obtain

$\left\{\ddot{\boldsymbol{p}}^{(1)}\right\}+\left(2 \frac{\ddot{a}}{a}-\Lambda\right)\left\{\boldsymbol{p}^{(1)}\right\}=\frac{\left\{\ddot{\boldsymbol{p}}^{(1)}\left(t_{\mathrm{o}}\right)\right\}}{a^{2}}$

and

$$
\begin{aligned}
\left\{\ddot{\boldsymbol{p}}^{(2)}\right\} & +\left(2 \frac{\ddot{a}}{a}-\Lambda\right)\left\{\boldsymbol{p}^{(2)}\right\}=\frac{\left\{\ddot{\boldsymbol{p}}^{(2)}\left(t_{\mathrm{o}}\right)\right\}}{a^{2}} \\
& -\frac{1}{a^{2}}\left[\frac{1}{2}(\ddot{a}-a \Lambda)\left\{\boldsymbol{p}^{(1)}, \boldsymbol{p}^{(1)}\right\}+a\left\{\ddot{\boldsymbol{p}}^{(1)}, \boldsymbol{p}^{(1)}\right\}\right] .
\end{aligned}
$$

For convenience, we choose $\delta \dot{\rho}^{(1)}=\delta \rho$ and $\delta \dot{\rho}^{(2)}=0$. The quadratic perturbation is chosen such that $\left\{\ddot{\boldsymbol{p}}^{(2)}\left(t_{\mathrm{o}}\right)\right\}=\boldsymbol{O}$ (cf. equation 6). (Recall that in the Lagrangian picture the density is not a dynamical variable; consequently, it need not be written in perturbed form.)

To solve equations (10), we use the first-order solutions given in B92, where it was shown that the solution of (10a) for longitudinal perturbations can be given in terms of three linearly independent, time-dependent functions, $q_{1}, q_{2}$ and $q_{p}$, and two vector functions of Lagrangian coordinates, $\boldsymbol{Q}_{1}$ and $\boldsymbol{Q}_{2}$, as follows (we drop the superscript $\mathrm{D}$ in this paper):

$\left\{\boldsymbol{p}^{(1)}\right\}=q_{1}\left\{\boldsymbol{Q}_{1}\right\}+q_{2}\left\{\boldsymbol{Q}_{2}\right\}+q_{p}\{\boldsymbol{P}\}$

where $P=-Q_{1}-Q_{2}$ specifies the choice of Lagrangian coordinates such that $\left\{\boldsymbol{p}^{(1)}\left(\boldsymbol{X}, t_{0}\right)\right\}=\boldsymbol{O}$ (for the equations the time-dependent functions must obey, see below).

Since the principal part of equation (10a) is identical to that of equation (10b), the homogeneous solution of equation (10b) has a similar form to the first-order solutions (11a) of (10a):

$\left\{\boldsymbol{p}_{\text {hom }}^{(2)}\right\}=q_{1}\left\{\boldsymbol{R}_{1}\right\}+q_{2}\left\{\boldsymbol{R}_{2}\right\}+q_{p}\left\{\boldsymbol{R}_{p}\right\}$.

The three quadratic coefficients $\boldsymbol{R}_{\ell}, \ell=1,2, p$, have to be determined in terms of the first-order coefficient functions $\boldsymbol{Q}_{1}$ and $\boldsymbol{Q}_{2}$ as follows. We compute the second-order part of the velocity $\left\{\dot{\boldsymbol{p}}^{(2)}\right\}$ and the acceleration $\left\{\ddot{\boldsymbol{p}}^{(2)}\right\}$ relative to the background (cf. Section 3.2) and obtain two equations for the quadratic coefficients by the requirement that the coefficient functions must vanish. The third equation is given by the requirement $\left\{\boldsymbol{p}^{(2)}\left(\boldsymbol{X}, t_{\mathrm{o}}\right)\right\}=\boldsymbol{0}$, which defines the Lagrangian coordinates according to our assumption (cf. equation 6). This is explicitly done in an example in Section 4. 


\section{T. Buchert and J. Ehlers}

In order to determine a particular solution of the secondorder equation (10b), we apply the formula

$$
\begin{aligned}
\left\{\boldsymbol{p}_{\mathrm{part}}^{(2)}(\boldsymbol{X}, t)\right\}= & \frac{1}{M}\left[q_{1}(t) \int^{t} \mathrm{~d} t q_{2}(t) \frac{\{\boldsymbol{G}(\boldsymbol{X}, t)\}}{a^{2}}\right. \\
& \left.-q_{2}(t) \int^{t} \mathrm{~d} t q_{1}(t) \frac{\{\boldsymbol{G}(\boldsymbol{X}, t)\}}{a^{2}}\right],
\end{aligned}
$$

with $\nabla_{\mathrm{o}} \cdot\{\boldsymbol{G}\}:=-\mathbb{Q}$, where

$$
\begin{aligned}
\{\boldsymbol{G}\}:= & \frac{1}{2}(\ddot{a}-a \Lambda)\left(q_{1}^{2}\left\{\boldsymbol{Q}_{1}, \boldsymbol{Q}_{1}\right\}+q_{2} q_{1}\left\{\boldsymbol{Q}_{2}, \boldsymbol{Q}_{1}\right\}+q_{p} q_{1}\left\{\boldsymbol{P}, \boldsymbol{Q}_{1}\right\}\right. \\
& +q_{1} q_{2}\left\{\boldsymbol{Q}_{1}, \boldsymbol{Q}_{2}\right\}+q_{2}^{2}\left\{\boldsymbol{Q}_{2}, \boldsymbol{Q}_{2}\right\}+q_{p} q_{2}\left\{\boldsymbol{P}, \boldsymbol{Q}_{2}\right\} \\
& \left.+q_{1} q_{p}\left\{\boldsymbol{Q}_{1}, \boldsymbol{P}\right\}+q_{2} q_{p}\left\{\boldsymbol{Q}_{2}, \boldsymbol{P}\right\}+q_{p}^{2}\{\boldsymbol{P}, \boldsymbol{P}\}\right) \\
& +a\left(\ddot{q}_{1} q_{1}\left\{\boldsymbol{Q}_{1}, \boldsymbol{Q}_{1}\right\}+\ddot{q}_{2} q_{1}\left\{\boldsymbol{Q}_{2}, \boldsymbol{Q}_{1}\right\}+\ddot{q}_{p} q_{1}\left\{\boldsymbol{P}, \boldsymbol{Q}_{1}\right\}\right. \\
& +\ddot{q}_{1} q_{2}\left\{\boldsymbol{Q}_{1}, \boldsymbol{Q}_{2}\right\}+\ddot{q}_{2} q_{2}\left\{\boldsymbol{Q}_{2}, \boldsymbol{Q}_{2}\right\}+\ddot{q}_{p} q_{2}\left\{\boldsymbol{P}, \boldsymbol{Q}_{2}\right\} \\
& \left.+\ddot{q}_{1} q_{p}\left\{\boldsymbol{Q}_{1}, \boldsymbol{P}\right\}+\ddot{q}_{2} q_{p}\left\{\boldsymbol{Q}_{2}, \boldsymbol{P}\right\}+\ddot{q}_{p} q_{p}\{\boldsymbol{P}, \boldsymbol{P}\}\right) .
\end{aligned}
$$

The constant $M$ is determined by inserting the full solution $\left\{\boldsymbol{p}^{(2)}\right\}=\left\{\boldsymbol{p}_{\text {hom }}^{(2)}\right\}+\left\{\boldsymbol{p}_{\text {part }}^{(2)}\right\}$ into equation $(10 \mathrm{~b})$.

\section{Theorem}

A large class of solutions for second-order Lagrangian irrotational perturbations at a homogeneous and isotropic background has the form

$\boldsymbol{f}^{Q}(\boldsymbol{X}, t)=a(t) \boldsymbol{X}+\varepsilon\left\{\boldsymbol{p}^{(1)}(\boldsymbol{X}, t)\right\}+\varepsilon^{2}\left\{\boldsymbol{p}^{(2)}(\boldsymbol{X}, t)\right\}$,

with

$$
\left\{\boldsymbol{p}^{(1)}(\boldsymbol{X}, t)\right\}=q_{1}(t)\left\{\boldsymbol{Q}_{1}(\boldsymbol{X})\right\}+q_{2}(t)\left\{\boldsymbol{Q}_{2}(\boldsymbol{X})\right\}+q_{p}(t)\{\boldsymbol{P}(\boldsymbol{X})\},
$$$$
\left\{\boldsymbol{p}^{(2)}(\boldsymbol{X}, t)\right\}=\left\{\boldsymbol{p}_{\text {hom }}^{(2)}(\boldsymbol{X}, t)\right\}+\left\{\boldsymbol{p}_{\text {part }}^{(2)}(\boldsymbol{X}, t)\right\}
$$

(cf. equations 11 ). The functions $q_{\ell}, \ell=1,2, p$, are linearly independent solutions of the first-order equation (10a) and obey the following equations:

$$
\begin{aligned}
& \left(2 \frac{\ddot{a}}{a}-\Lambda\right) q_{\ell}+\ddot{q}_{\ell}=0, \quad \ell=1,2 ; \\
& \left(2 \frac{\ddot{a}}{a}-\Lambda\right) q_{p}+\ddot{q}_{p}=\left[\ddot{q}_{p}\left(t_{\mathrm{o}}\right)-\ddot{q}_{\ell}\left(t_{\mathrm{o}}\right)\right] a^{-2}, \quad \ell=1,2
\end{aligned}
$$

(see B92). All coefficient functions that depend on Lagrangian coordinates are fully determined by the longitudinal coefficient functions $\left\{Q_{1}\right\}=: \nabla_{\mathrm{o}} \Omega_{1},\left\{\boldsymbol{Q}_{2}\right\}=: \nabla_{\mathrm{o}} \Omega_{2}$ of the firstorder solutions. In general, they are not expressible in closed form and have to be calculated from initial conditions by solving elliptic boundary value problems (cf. equations 8). (An exception is presented in the following Corollary.) The generality of the solution with respect to the whole class of irrotational flows is restricted by the functional relationship $\nabla_{\mathrm{o}} \Omega_{2}=\mathscr{H}\left(\nabla_{\mathrm{o}} \Omega_{1}\right)$, with arbitrary $\mathscr{H}$.

To prove this theorem, we have to insert the solution into equations (10). (Note: the equations that the time-dependent functions obey have been corrected; see the Erratum to B92 given in Appendix B.) In general, two scalar functions of three variables (e.g. the velocity potential and the gravitational potential) can be given independently for irrotational perturbations. Insertion of the solution into the constraint equations $(5 \mathrm{~d}, \mathrm{e}, \mathrm{f})$ and retention of terms up to the second order shows that the gradients of the two initial potentials $\Omega_{1}$ and $\Omega_{2}$, or the velocity potential and the gravitational potential, respectively, have to be functionally dependent. This is (apart from irrotationality) the only restriction on initial conditions. (The constraint equations are listed in Appendix A.)

Q.e.d.

Remarks. For most applications, the above-mentioned restriction is demanded anyway (see Section 5). The splitting of the perturbation function into a transverse part and a longitudinal part implies no restriction of generality. The non-uniqueness of this splitting expresses the fact that we have some gauge freedom in deriving the solutions. Representations of the solution that are different from the one presented might exist.

Corollary 1

The following class of second-order solutions can be written in closed form. This class is obtained by replacing the general vector fields defined in (9) by special vector fields as follows. For arbitrary vector fields $\boldsymbol{A}, \boldsymbol{B}, \boldsymbol{C}$, we perform the replacements

$$
\begin{aligned}
\{\boldsymbol{A}\} \rightarrow \boldsymbol{A}, & \\
\{\boldsymbol{A}, \boldsymbol{A}\} \rightarrow & \boldsymbol{A}\left(\nabla_{\mathrm{o}} \cdot \boldsymbol{A}\right)-\left(\boldsymbol{A} \cdot \nabla_{\mathrm{o}}\right) \boldsymbol{A}, \\
\{\boldsymbol{B}, \boldsymbol{C}\} \rightarrow & \lambda_{1}\left[\boldsymbol{B}\left(\nabla_{\mathrm{o}} \cdot \boldsymbol{C}\right)-\left(\boldsymbol{B} \cdot \nabla_{\mathrm{o}}\right) \boldsymbol{C}\right]+\lambda_{2}\left[\boldsymbol{C}\left(\nabla_{\mathrm{o}} \cdot \boldsymbol{B}\right)-\left(\boldsymbol{C} \cdot \nabla_{\mathrm{o}}\right) \boldsymbol{B}\right], \\
& \lambda_{1}+\lambda_{2}=1 .
\end{aligned}
$$

We then have, from $(9)$,

$$
\begin{aligned}
& \nabla_{\mathrm{o}} \cdot \boldsymbol{A}=I(\boldsymbol{A}), \quad \nabla_{\mathrm{o}} \times \boldsymbol{A}=\boldsymbol{0} \\
& \nabla_{\mathrm{o}} \cdot\left[\boldsymbol{A}\left(\nabla_{\mathrm{o}} \cdot \boldsymbol{A}\right)-\left(\boldsymbol{A} \cdot \nabla_{\mathrm{o}}\right) \boldsymbol{A}\right]=2 \cdot \boldsymbol{I I}(\boldsymbol{A}), \\
& \nabla_{\mathrm{o}} \times\left[\boldsymbol{A}\left(\nabla_{\mathrm{o}} \cdot \boldsymbol{A}\right)-\left(\boldsymbol{A} \cdot \nabla_{\mathrm{o}}\right)\right]=\boldsymbol{A} \times \Delta \boldsymbol{A} \\
& \nabla_{\mathrm{o}} \cdot \lambda_{1}\left[\boldsymbol{B}\left(\nabla_{\mathrm{o}} \cdot \boldsymbol{C}\right)-\left(\boldsymbol{B} \cdot \nabla_{\mathrm{o}}\right) \boldsymbol{C}\right]+\lambda_{2}\left[\boldsymbol{C}\left(\nabla_{\mathrm{o}} \cdot \boldsymbol{B}\right)-\left(\boldsymbol{C} \cdot \nabla_{\mathrm{o}}\right) \boldsymbol{B}\right] \\
& =\sum_{a, b, c} \epsilon_{a b c} \frac{\partial\left(B_{a}, C_{b}, X_{c}\right)}{\partial\left(X_{1}, X_{2}, X_{3}\right)} \\
& \nabla_{\mathrm{o}} \times\left(\lambda_{1}\left[\boldsymbol{B}\left(\nabla_{\mathrm{o}} \cdot \boldsymbol{C}\right)-\left(\boldsymbol{B} \cdot \nabla_{\mathrm{o}}\right) \boldsymbol{C}\right]+\lambda_{2}\left[\boldsymbol{C}\left(\nabla_{\mathrm{o}} \cdot \boldsymbol{B}\right)-\left(\boldsymbol{C} \cdot \nabla_{\mathrm{o}}\right) \boldsymbol{B}\right]\right) \\
& =\lambda_{1}\left(B \times \Delta_{\mathrm{o}} \boldsymbol{C}\right)+\lambda_{2}\left(\boldsymbol{C} \times \Delta_{\mathrm{o}} \boldsymbol{B}\right) .
\end{aligned}
$$

Thus the potential property of the flow is preserved if the following constraints are satisfied:

$$
\begin{array}{ll}
\nabla_{\mathrm{o}} \times \boldsymbol{A}=\boldsymbol{0}, & \nabla_{\mathrm{o}} \times \boldsymbol{B}=\boldsymbol{0}, \quad \nabla_{\mathrm{o}} \times \boldsymbol{C}=\mathbf{0} \\
\boldsymbol{A} \times \Delta_{\mathrm{o}} \boldsymbol{A}=\boldsymbol{0}, & \lambda_{1}\left(\boldsymbol{B} \times \Delta_{\mathrm{o}} \boldsymbol{C}\right)+\lambda_{2}\left(\boldsymbol{C} \times \Delta_{\mathrm{o}} \boldsymbol{B}\right)=\boldsymbol{0} .
\end{array}
$$

This Corollary tells us that certain additional constraints have to be fulfilled to assure that the second-order solutions that include the replacements still provide irrotational flows. This is non-trivial, since vector functions of the form $\boldsymbol{A}\left(\nabla_{\mathrm{o}} \cdot \boldsymbol{A}\right)-\left(\boldsymbol{A} \cdot \nabla_{\mathrm{o}}\right) \boldsymbol{A}$ are not curl-free in Lagrangian space. In general, these terms also introduce vorticity in Eulerian space. For special initial conditions, however, the terms have zero vorticity. In this case, the solutions preserve the potential property of the velocity field, which can be demonstrated by computing the vorticity with the help of equations $(5 a, b, c)$ using the constraints given in Corollary 1 . Note that, initially, the vorticity in Lagrangian space has to vanish. If it does not, well-known vorticity theorems show that the potential property is destroyed.

The advantage of the replacements defined in Corollary 1 for the local description of perturbations has already been demonstrated in the first-order solutions and their applica- 
tions (see B92): we can directly map any generic field without explicitly solving the Poisson equations (8) for given initial conditions. In the second-order case, this advantage can (for generic initial conditions) only be obtained at the cost of vorticity generation. A more detailed discussion of this issue is given in Section 5 .

\subsection{The Lagrangian theory in a frame comoving with the} background

For application of the perturbative solutions as models for the formation of large-scale structure, we define the 'comoving Eulerian frame' and the 'peculiar fields' as comoving deviations from the Hubble velocity and the Hubble acceleration. This corresponds to a choice of Eulerian coordinates $\boldsymbol{q}:=\boldsymbol{x} / a(t)$. In the Lagrangian picture, we introduce the 'comoving' orbit $\boldsymbol{F}$ :

$$
\begin{gathered}
\boldsymbol{q}=\boldsymbol{F}(\boldsymbol{X}, t):=\frac{1}{a(t)} \boldsymbol{f}(\boldsymbol{X}, t)=\boldsymbol{X}+\frac{\boldsymbol{p}(\boldsymbol{X}, t)}{a(t)}, \\
\boldsymbol{p}=\varepsilon \boldsymbol{p}^{(1)}+\varepsilon^{2} \boldsymbol{p}^{(2)} .
\end{gathered}
$$

All fields continue to be represented in the same Lagrangian frame $\boldsymbol{X}$ if we define $a\left(t_{\mathrm{o}}\right):=1$ (equation 6). In this comoving picture, we introduce the peculiar velocity $\boldsymbol{u}$ and the peculiar acceleration $\boldsymbol{w}$ as usual:

$\boldsymbol{v}=\dot{\boldsymbol{f}}=\dot{a} \boldsymbol{F}+\boldsymbol{u}, \quad \boldsymbol{u}:=a \dot{\boldsymbol{F}}$

$\boldsymbol{g}=\ddot{\boldsymbol{f}}=\ddot{a} \boldsymbol{F}+w, \quad w:=2 \dot{a} \dot{\boldsymbol{F}}+a \ddot{\boldsymbol{F}}=\dot{\boldsymbol{u}}+\frac{\dot{a}}{a} \boldsymbol{u}$

Note that the convective derivative with respect to $v$ is equivalent to the convective derivative with respect to $\boldsymbol{u} / a$ in the comoving picture:

$\frac{\mathrm{d}}{\mathrm{d} t}:=\left.\partial_{t}\right|_{x}+v \cdot \nabla_{x}=\left.\partial_{t}\right|_{q}+\frac{u}{a} \nabla_{q}$

\section{EXAMPLE: A PANCAKE MODEL FOR SECOND-ORDER IRROTATIONAL PERTURBATIONS OF A FLAT UNIVERSE}

In this example, we present a large class of second-order solutions for inhomogeneous irrotational deformations of a flat background universe. Setting (constant $=0 ; \Lambda=0)$ in equation $(7 \mathrm{~b})$, we obtain

$a(t)=\left(\frac{t}{t_{\mathrm{o}}}\right)^{2 / 3}$

With the ansatz $q_{\ell}(t)=\left(t / t_{\mathrm{o}}\right)^{n}, n=1,2, p$, we seek solutions for the time-dependent functions in the homogeneous solutions $\left\{\boldsymbol{p}_{\text {hom }}^{(1)}\right\}$ and $\left\{\boldsymbol{p}_{\text {hom }}^{(2)}\right\}$. We obtain

$n_{1}=\frac{4}{3}, \quad n_{2}=-\frac{1}{3}, \quad n_{p}=\frac{2}{3}$

(see B92). The linear inhomogeneous deformation $\left\{\boldsymbol{p}^{(1)}\right\}$ reads

$\left\{\boldsymbol{p}^{(1)}\right\}=\left(\frac{t}{t_{\mathrm{o}}}\right)^{4 / 3}\left\{\boldsymbol{Q}_{1}(\boldsymbol{X})\right\}+\left(\frac{t}{t_{\mathrm{o}}}\right)^{-1 / 3}\left\{\boldsymbol{Q}_{2}(\boldsymbol{X})\right\}+\left(\frac{t}{t_{\mathrm{o}}}\right)^{2 / 3}\{\boldsymbol{P}(\boldsymbol{X})\}$
(B92). Using (16), we can express the initial perturbation fields in terms of the initial conditions for the peculiar velocity $\boldsymbol{U}:=\boldsymbol{u}\left(\boldsymbol{X}, t_{\mathrm{o}}\right)$ and the peculiar acceleration $\boldsymbol{W}:=\boldsymbol{w}\left(\boldsymbol{X}, t_{\mathrm{o}}\right)$ as follows:

$\left\{\boldsymbol{Q}_{1}\right\}=\frac{3}{5}\{\boldsymbol{U}\} t_{\mathrm{o}}+\frac{9}{10} \boldsymbol{W} t_{\mathrm{o}}^{2}$

$\left\{\boldsymbol{Q}_{2}\right\}=-\frac{3}{5}\{\boldsymbol{U}\} t_{\mathrm{o}}+\frac{3}{5} \boldsymbol{W} t_{\mathrm{o}}^{2}$

$\{\boldsymbol{P}\}=-\left\{\boldsymbol{Q}_{1}\right\}-\left\{\boldsymbol{Q}_{2}\right\}=-\frac{3}{2} \boldsymbol{W} t_{\mathrm{o}}^{2}$

(B92). According to (11b), the homogeneous solution of the second-order perturbation can be written

$\left\{\boldsymbol{p}_{\text {hom }}^{(2)}\right\}=\left(\frac{t}{t_{\mathrm{o}}}\right)^{4 / 3}\left\{\boldsymbol{R}_{1}(\boldsymbol{X})\right\}+\left(\frac{t}{t_{\mathrm{o}}}\right)^{-1 / 3}\left\{\boldsymbol{R}_{2}(\boldsymbol{X})\right\}+\left(\frac{t}{t_{\mathrm{o}}}\right)^{2 / 3}\left\{\boldsymbol{R}_{p}(\boldsymbol{X})\right\}$

We now evaluate the particular solution $\left\{\boldsymbol{p}_{\text {part }}^{(2)}\right\}$. We have to calculate the time-dependent coefficient functions of the quadratic coefficients in (11c). We denote these by

$z_{Q_{1}, Q_{1}}=\frac{1}{3 t_{\mathrm{o}}^{2}}\left(\frac{t}{t_{\mathrm{o}}}\right)^{r_{Q_{1}, Q_{1}}}, \quad$ etc....

For the power indices $r$, we obtain

$r_{Q_{1}, Q_{1}}=\frac{4}{3}, \quad r_{Q_{1}, Q_{2}}=-\frac{1}{3}, \quad r_{Q_{1}, P}=\frac{2}{3}$,

$r_{Q_{2}, Q_{1}}=-\frac{1}{3}, \quad r_{Q_{2}, Q_{2}}=-2, \quad r_{Q_{2}, P}=-1$,

$r_{P, Q_{1}}=\frac{2}{3}, \quad r_{P, Q_{2}}=-1, \quad r_{P, P}=0$

After integration (cf. equation 11c), and determination of the integration constant $M$ to $M=-5 /\left(3 t_{\mathrm{o}}\right)$, we obtain

$$
\begin{aligned}
\left\{\boldsymbol{p}_{\text {part }}^{(2)}\right\}= & -\frac{3}{14}\left\{\boldsymbol{Q}_{1}, \boldsymbol{Q}_{1}\right\}\left(\frac{t}{t_{\mathrm{o}}}\right)^{2}+\frac{1}{2}\left(\left\{\boldsymbol{Q}_{1}, \boldsymbol{Q}_{2}\right\}\right. \\
& \left.+\left\{\boldsymbol{Q}_{2}, \boldsymbol{Q}_{1}\right\}\right)\left(\frac{t}{t_{\mathrm{o}}}\right)^{1 / 3}-\frac{1}{8}\left\{\boldsymbol{Q}_{2}, \boldsymbol{Q}_{2}\right\}\left(\frac{t}{t_{\mathrm{o}}}\right)^{-4 / 3} .
\end{aligned}
$$

From this solution, together with the homogeneous parts $\left\{\boldsymbol{p}_{\text {hom }}^{(1)}\right\}$ and $\left\{\boldsymbol{p}_{\text {hom }}^{(2)}\right\}$, we compute the initial velocity and acceleration relative to the Hubble flow and determine the quadratic coefficients of the homogeneous second-order solution (18) to

$$
\begin{aligned}
& \left\{\boldsymbol{R}_{1}\right\}=\frac{3}{5}\left\{\boldsymbol{Q}_{1}, \boldsymbol{Q}_{1}\right\}+\frac{1}{10}\left(\left\{\boldsymbol{Q}_{1}, \boldsymbol{Q}_{2}\right\}+\left\{\boldsymbol{Q}_{2}, \boldsymbol{Q}_{1}\right\}\right)+\frac{9}{40}\left\{\boldsymbol{Q}_{2}, \boldsymbol{Q}_{2}\right\}, \\
& \left\{\boldsymbol{R}_{2}\right\}=\frac{4}{35}\left\{\boldsymbol{Q}_{1}, \boldsymbol{Q}_{1}\right\}-\frac{1}{10}\left(\left\{\boldsymbol{Q}_{1}, \boldsymbol{Q}_{2}\right\}+\left\{\boldsymbol{Q}_{2}, \boldsymbol{Q}_{1}\right\}\right)+\frac{2}{5}\left\{\boldsymbol{Q}_{2}, \boldsymbol{Q}_{2}\right\},
\end{aligned}
$$

\section{Royal Astronomical Society - Provided by the NASA Astrophysics Data System}




$$
\begin{aligned}
\left\{\boldsymbol{R}_{p}\right\}= & -\frac{1}{2}\{\boldsymbol{P}, \boldsymbol{P}\}=-\frac{1}{2}\left\{\boldsymbol{Q}_{1}, \boldsymbol{Q}_{1}\right\}-\frac{1}{2}\left(\left\{\boldsymbol{Q}_{1}, \boldsymbol{Q}_{2}\right\}\right. \\
& \left.+\left\{\boldsymbol{Q}_{2}, \boldsymbol{Q}_{1}\right\}\right)-\frac{1}{2}\left\{\boldsymbol{Q}_{2}, \boldsymbol{Q}_{2}\right\}
\end{aligned}
$$

We finally write down the full solution $f^{Q}$ in terms of the initial vector functions $\boldsymbol{Q}_{1}$ and $\boldsymbol{Q}_{2}$, which can be related to the initial conditions for the peculiar velocity and peculiar acceleration according to (17); henceforth, $\varepsilon$ is assumed to be absorbed into the amplitudes of the initial conditions:

$$
\begin{aligned}
\boldsymbol{f}^{Q}(\boldsymbol{X}, t)= & \left(\frac{t}{t_{\mathrm{o}}}\right)^{2 / 3} \boldsymbol{X}+\left(\frac{t}{t_{\mathrm{o}}}\right)^{4 / 3}\left\{\boldsymbol{Q}_{1}\right\}+\left(\frac{t}{t_{\mathrm{o}}}\right)^{-1 / 3}\left\{\boldsymbol{Q}_{2}\right\} \\
& +\left(\frac{t}{t_{\mathrm{o}}}\right)^{2 / 3}\left(-\left\{\boldsymbol{Q}_{1}\right\}-\left\{\boldsymbol{Q}_{2}\right\}\right)+\left(\frac{t}{t_{\mathrm{o}}}\right)^{2}\left(-\frac{3}{14}\left\{\boldsymbol{Q}_{1}, \boldsymbol{Q}_{1}\right\}\right) \\
& +\left(\frac{t}{t_{\mathrm{o}}}\right)^{4 / 3}\left[\frac{3}{5}\left\{\boldsymbol{Q}_{1}, \boldsymbol{Q}_{1}\right\}+\frac{1}{10}\left(\left\{\boldsymbol{Q}_{1}, \boldsymbol{Q}_{2}\right\}+\left\{\boldsymbol{Q}_{2}, \boldsymbol{Q}_{1}\right\}\right)\right. \\
& \left.+\frac{9}{40}\left\{\boldsymbol{Q}_{2}, \boldsymbol{Q}_{2}\right\}\right]+\left(\frac{t}{t_{\mathrm{o}}}\right)^{2 / 3}\left[-\frac{1}{2}\left\{\boldsymbol{Q}_{1}, \boldsymbol{Q}_{1}\right\}\right. \\
& \left.-\frac{1}{2}\left(\left\{\boldsymbol{Q}_{1}, \boldsymbol{Q}_{2}\right\}+\left\{\boldsymbol{Q}_{2}, \boldsymbol{Q}_{1}\right\}\right)-\frac{1}{2}\left\{\boldsymbol{Q}_{2}, \boldsymbol{Q}_{2}\right\}\right] \\
& +\left(\frac{t}{t_{\mathrm{o}}}\right)^{1 / 3}\left[\frac{1}{2}\left(\left\{\boldsymbol{Q}_{1}, \boldsymbol{Q}_{2}\right\}+\left\{\boldsymbol{Q}_{2}, \boldsymbol{Q}_{1}\right\}\right)\right] \\
& +\left(\frac{t}{t_{\mathrm{o}}}\right)^{-1 / 3}\left[\frac{4}{35}\left\{\boldsymbol{Q}_{1}, \boldsymbol{Q}_{1}\right\}-\frac{1}{10}\left(\left\{\boldsymbol{Q}_{1}, \boldsymbol{Q}_{2}\right\}+\left\{\boldsymbol{Q}_{2}, \boldsymbol{Q}_{1}\right\}\right)\right. \\
& \left.+\frac{2}{5}\left\{\boldsymbol{Q}_{2}, \boldsymbol{Q}_{2}\right\}\right]+\left(\frac{t}{t_{\mathrm{o}}}\right)^{-4 / 3}\left(-\frac{1}{8}\left\{\boldsymbol{Q}_{2}, \boldsymbol{Q}_{2}\right\}\right) .
\end{aligned}
$$

From the general solution $(2 \mathrm{~d})$, the density is given by:

$\rho(\boldsymbol{X}, t)=\stackrel{\rho}{ }(\boldsymbol{X})\left(\operatorname{det}\left[f_{i, k}^{Q}(\boldsymbol{X}, t)\right]\right)^{-1}$.

The initial conditions $\stackrel{\rho}{\rho}=\circ_{\mathrm{H}}-[1 /(4 \pi G)] \nabla_{\mathrm{o}} \cdot \boldsymbol{W}, \boldsymbol{W}=:-\nabla_{\mathrm{o}} \phi$ and $\boldsymbol{U}=: \nabla_{\mathrm{o}} \mathscr{S}$ can be expressed in terms of two functions of three variables (the peculiar-gravity potential $\phi$ and the peculiar-velocity potential $\mathscr{S}$ ), which can be given independently, if the solution is general. The constraint equations from the integrability conditions $(5 \mathrm{~d}, \mathrm{e}, \mathrm{f})$ introduce the following additional relation which has to be fulfilled:

$\nabla_{\mathrm{o}} \phi=\mathscr{F}\left(\nabla_{\mathrm{o}} \mathscr{S}\right)$.

In particular, relation (22c) implies $\nabla_{\mathrm{o}} \Omega_{2}=\mathscr{H}\left(\nabla_{\mathrm{o}} \Omega_{1}\right)$, which simplifies the general form (22a). Special models using restricted initial conditions that satisfy $(22 c)$ are discussed in Section 5.

\section{DISCUSSION AND ILLUSTRATION}

5.1 Restricted models as generalizations of Zel'dovich's approximation

In the discussion of the first-order solutions in B92, we looked at different possibilities to restrict the solutions such that only one initial field has to be given. Two such possible restrictions were considered to represent Zel'dovich's approximation (Zel'dovich 1970, 1973). In this spirit, we now define second-order models with similar restrictions on initial data.

One possibility to restrict solutions $(22)$ is to require that

$\left\{\boldsymbol{Q}_{1}\right\}=-\left\{\boldsymbol{Q}_{2}\right\} \Leftrightarrow \boldsymbol{W}(\boldsymbol{X})=\boldsymbol{0} ; \quad \mathscr{F}=0$.

Inserting (23a) into the general orbit (22), we obtain

$$
\begin{aligned}
\boldsymbol{F}_{Z 1}^{Q}(\boldsymbol{X}, t)= & \boldsymbol{X}+\left[\left(\frac{t}{t_{\mathrm{o}}}\right)^{2 / 3}-\left(\frac{t}{t_{\mathrm{o}}}\right)^{-1}\right] \frac{3}{5}\{\boldsymbol{U}(\boldsymbol{X})\} t_{\mathrm{o}} \\
& +\left[-\frac{3}{14}\left(\frac{t}{t_{\mathrm{o}}}\right)^{4 / 3}+\frac{29}{40}\left(\frac{t}{t_{\mathrm{o}}}\right)^{2 / 3}-\frac{1}{2}-\frac{1}{2}\left(\frac{t}{t_{\mathrm{o}}}\right)^{-1 / 3}\right. \\
& \left.+\frac{43}{70}\left(\frac{t}{t_{\mathrm{o}}}\right)^{-1}-\frac{1}{8}\left(\frac{t}{t_{\mathrm{o}}}\right)^{-2}\right] \frac{9}{25}\{\boldsymbol{U}(\boldsymbol{X}), \boldsymbol{U}(\boldsymbol{X})\} t_{\mathrm{o}}^{2}
\end{aligned}
$$

i.e. the density is initially given by the homogeneous background density, and the fluctuations are produced solely by velocity perturbations.

A second possibility is to require the following condition to hold at the initial time:

$$
\left\{\boldsymbol{Q}_{2}\right\}=\boldsymbol{O} \Leftrightarrow\{\boldsymbol{U}(\boldsymbol{X})\}=\boldsymbol{W}(\boldsymbol{X}) t_{\mathrm{o}} ; \quad \mathscr{F}=\frac{i d}{t_{\mathrm{o}}},
$$

This relation between the initial peculiar-velocity field and the initial peculiar-acceleration field implicitly holds in Zel'dovich's ansatz. Initially, there is a density perturbation proportional to the velocity perturbation. The following physical argument prefers this assumption to restriction (23a): calculating the asymptotic large-time behaviour of the orbits $(22 \mathrm{a})$, i.e. neglecting the decaying solutions in the deformation field, we find that (up to a constant displacement vector of order $\left.\varepsilon^{2}\right)$ the peculiar velocity is related to the peculiar acceleration as $\{\boldsymbol{u}\}=\boldsymbol{w} t$. Thus at some sufficiently late time $t_{S}$ the two fields tend to be parallel, and we practically have $\left\{\boldsymbol{u}\left(\boldsymbol{X}, t_{s}\right)\right\}=\boldsymbol{w}\left(\boldsymbol{X}, t_{s}\right) t_{s}$. This corresponds to assumption (24a) for the initial conditions. We stress, however, that relaxing of constraint (22c) might destroy this property for independent initial conditions. The same is true for rotational initial conditions. Inserting (24a) into the general orbit (22), we obtain an extension of Zel'dovich's mapping into the second-order regime:

$$
\begin{aligned}
\boldsymbol{F}_{Z 2}^{Q}(\boldsymbol{X}, t)= & \boldsymbol{X}+\left[\left(\frac{t}{t_{\mathrm{o}}}\right)^{2 / 3}-1\right] \frac{3}{2}\{\boldsymbol{U}(\boldsymbol{X})\} t_{\mathrm{o}} \\
& +\left[-\frac{3}{14}\left(\frac{t}{t_{\mathrm{o}}}\right)^{4 / 3}+\frac{3}{5}\left(\frac{t}{t_{\mathrm{o}}}\right)^{2 / 3}-\frac{1}{2}+\frac{4}{35}\left(\frac{t}{t_{\mathrm{o}}}\right)^{-1}\right] \\
& \times \frac{9}{4}\{\boldsymbol{U}(\boldsymbol{X}), \boldsymbol{U}(\boldsymbol{X})\} t_{\mathrm{o}}^{2} .
\end{aligned}
$$

[For a related discussion of restrictions (23) and (24), see Buchert (1989), section 4.2.1, and B92.] Note that the constants, e.g. $-1 / 2$ in equation (24b), can simply be transformed away by introducing a different Lagrangian frame 
(see B92). Here we use a Lagrangian frame that coincides with a rectangular grid of the background at the initial time $t_{\mathrm{o}}$. This is useful because, for example, the initial conditions can be given on an undeformed grid.

In general, the initial conditions have to be calculated by solving Poisson equations. The mapping (24b), for example, can be written in terms of the peculiar-velocity potential $\mathscr{S}$ as follows (note that $\nabla_{\mathrm{o}} \phi=-\nabla_{\mathrm{o}} \mathscr{S} t_{\mathrm{o}}$ in this case):

$$
\begin{aligned}
\boldsymbol{F}_{Z 2}^{Q}(\boldsymbol{X}, t)= & \boldsymbol{X}+\left[\left(\frac{t}{t_{\mathrm{o}}}\right)^{2 / 3}-1\right] \frac{3}{2} \nabla_{\mathrm{o}} \mathscr{S}^{(1)}(\boldsymbol{X}) t_{\mathrm{o}} \\
& +\left[-\frac{3}{14}\left(\frac{t}{t_{\mathrm{o}}}\right)^{4 / 3}+\frac{3}{5}\left(\frac{t}{t_{\mathrm{o}}}\right)^{2 / 3}-\frac{1}{2}+\frac{4}{35}\left(\frac{t}{t_{\mathrm{o}}}\right)^{-1}\right] \\
& \times \frac{9}{4} \nabla_{\mathrm{o}} \mathscr{S}^{(2)}(\boldsymbol{X}) t_{\mathrm{o}}^{2},
\end{aligned}
$$

in which the first- and second-order peculiar potentials are to be solutions of Poisson equations, the sources of which are calculated iteratively with the initial potential $\mathscr{S}(\boldsymbol{X})$ (cf. equations 8):

$\Delta_{\mathrm{o}} \mathscr{S}^{(1)}=\sum_{i} \mathscr{S}_{, i, i}^{(1)}=I\left(\mathscr{S}_{, i, k}\right)$

$\Delta_{\mathrm{o}} \mathscr{S}^{(2)}=\left[\left(\sum_{i} \mathscr{S}_{, i, i}^{(1)}\right)^{2}-\sum_{i k}\left(\mathscr{S}_{, i, k}^{(1)} \mathscr{S}_{, k, i}^{(1)}\right)\right]=2 I I\left(\mathscr{S}_{, i, k}^{(1)}\right)$.

According to Corollary 1 , a simplification can be obtained in the special case in which $\nabla_{\mathrm{o}} \mathscr{S}^{(1)}$ and $\nabla_{\mathrm{o}} \mathscr{S}^{(2)}$ can be written in a closed form that is directly dependent on the initial potential $\mathscr{S}(\boldsymbol{X})$ :

$$
\begin{aligned}
& \nabla_{\mathrm{o}} \mathscr{S}^{(1)}=\nabla_{\mathrm{o}} \mathscr{S} ; \\
& \nabla_{\mathrm{o}} \mathscr{S}^{(2)}=\nabla_{\mathrm{o}} \mathscr{S}\left(\Delta_{\mathrm{o}} \mathscr{S}\right)-\left(\nabla_{\mathrm{o}} \mathscr{S} \cdot \nabla_{\mathrm{o}}\right) \nabla_{\mathrm{o}} \mathscr{S} ; \\
& \nabla_{\mathrm{o}} \mathscr{S} \times \Delta_{\mathrm{o}} \nabla_{\mathrm{o}} \mathscr{S}=\mathbf{O} .
\end{aligned}
$$

In the first Poisson equation, the use of $\mathscr{S}$ instead of $\mathscr{S}^{(1)}$ implies no restriction of generality in the sense that the addition of harmonic functions $\psi, \Delta_{\mathrm{o}} \psi=0$ will not affect physical quantities such as the density field. The choice of $\psi$ corresponds to the gauge freedom explained in Section 3 (Remarks). In the second Poisson equation, however, replacement (26b) cannot be used without loss of generality. Nevertheless, it turns out that the simplified form (26b) can serve as a good approximation even for generic initial conditions. This helps to circumvent the use of Poisson solvers (see Section 5.3).

\subsection{General remarks on properties of the solutions}

In the following, we make some remarks on the implications of the theorem for properties of the solutions presented.

\section{Corollary 2}

The flow field for the presented class of second-order Lagrangian irrotational perturbations is curl-free with respect to the Lagrangian frame.

The proof follows from the form of the solutions (theorem), using the definition equation (1) for the integral curves of the velocity field.

Q.e.d.
Although, in general, the irrotationality with respect to the Lagrangian frame is more restrictive than the irrotationality with respect to the Eulerian frame, this implies (besides the functional dependence 22c) no further restriction of the generality of the solutions, since we obtain a solution for any initial velocity potential. The stronger condition of irrotationality in Lagrangian space might not hold for general irrotational second-order flows. Moreover, it might also not hold if the solutions are non-separable with respect to Lagrangian coordinates and time, a property that is expected for the general exact solution. In the case of the perturbation theory, the solutions are separable by construction.

Corollary 3

The singularities of the presented solutions are Lagrangian.

Proof: it has been demonstrated in B92 that the first-order solutions for irrotational perturbations can be considered as a family of Lagrangian mappings from Lagrangian to Eulerian space. We now show that the presented class of solutions does not destroy the Lagrangian property.

Recall that the motion of the fluid naturally describes a manifold as the three-dimensional hypersurface in sixdimensional phase space that is generated by the flow, i.e. by the collection of all trajectories. Let us introduce the Lagrangian coordinates $X_{i}$ as local coordinates on this manifold, and the Eulerian coordinates as local coordinates in real space. The family of mappings

$\pi_{t}: \mathbb{R}^{3} \rightarrow \mathbb{R}^{3}, \quad \boldsymbol{X} \mapsto \boldsymbol{x}=\boldsymbol{f}^{Q}(\boldsymbol{X} ; t)$

defines a family of Lagrangian mappings if the two-form $\Omega=\Sigma_{i} \mathrm{~d} x_{i} \wedge \mathrm{d} X_{i}$ vanishes on the manifold $\pi_{t}$. In that case, the manifold generated by the flow lines is Lagrangian according to Arnol'd's theory (Arnol'd, Gusein-Zade \& Varchenko 1985). This family of mappings specialized to the first order is Lagrangian, since it can be written as a family of gradients $\boldsymbol{x}=\nabla_{\mathrm{o}} \mathrm{Y}(\boldsymbol{X} ; t)$ (see $\mathrm{B} 92$ ), which implies vanishing of the form $\Omega$. The same can be said for mapping (22a), because the second-order coefficients of the longitudinal part of the perturbations also have zero vorticity in Lagrangian space according to Corollary $2 . \quad$ Q.e.d.

Note that in the Lagrangian perturbation approach the solutions separate with respect to Lagrangian coordinates and time by construction. Thus the above consideration applies to any order of the perturbation theory as far as the Lagrangian longitudinal part of the perturbations is concerned. We stress, however, that the general mapping (which might not separate with respect to Lagrangian coordinates and time) might be non-Lagrangian, and the perturbation expansion might not converge to the general solution. Also, relaxation of constraints $(22 \mathrm{c})$ might introduce vorticity in Lagrangian space.

\subsection{Illustration}

We take as an illustration the special solutions (24c) as an extension of Zel'dovich's mapping into the second-order regime, and restrict the problem to two spatial dimensions. We present a realization of a particular density field (mapped by $200^{2}$ particles). We use as the initial condition for the peculiar-velocity potential $\mathscr{S}$ the following periodic function, which can be regarded as illustrating principal features of the 


\section{T. Buchert and J. Ehlers}

topology of the large-scale structure out of a truncated power spectrum:

$\mathscr{S}=-\frac{1}{2} \frac{\mu}{(2 \pi n)^{2}} \cos (2 \pi n X) \cos (2 \pi n Y), \quad n \in \mathbb{N}$

(a)

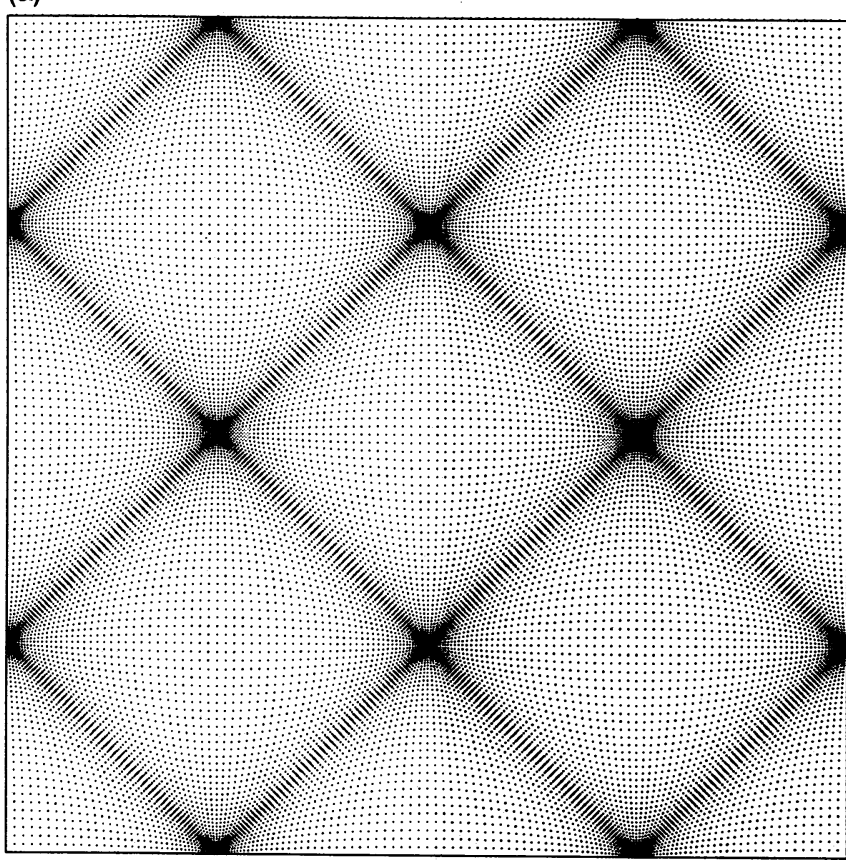

(b)
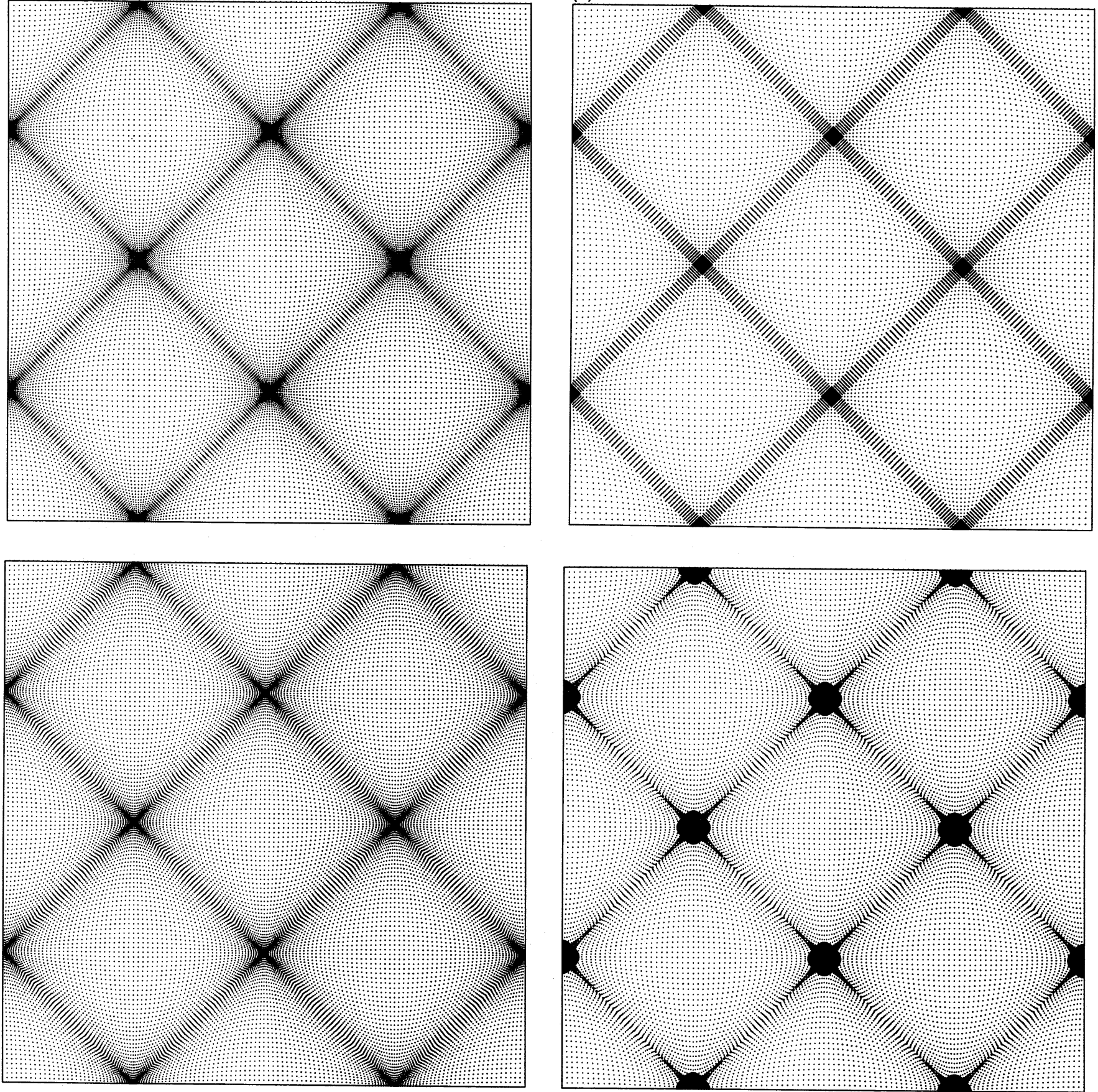

Figure 1. Four redshift panels, $Z=1,0.5,0.25$ and 0 , are shown in (a), (b), (c) and (d), respectively, for the first-order approximation (the 'Zel'dovich approximation' in this case) (upper plots) and the second-order approximation (lower plots). The initial condition is a special periodic function that maps principal elements of the large-scale structure such as sheets and clusters. At a stage shortly after the first shellcrossing (in this normalization at $z=1)\left(\sigma_{\mathrm{o}}=2.0\right)$, the two approximations give similar results, except for a slight departure from the purely onedimensional infall on to sheets (as in the first-order case) orientated towards the clusters. The clusters themselves appear more compact and the sheets much thinner in the second-order approximation, even at much later stages. 
sian random fluctuations:

$\mathscr{S}=\mu \sum_{k_{\min }}^{k_{\max }} \sum_{k_{\min }}^{k_{\max }} \frac{1}{|k|^{2}}\{A(\boldsymbol{k}) \cos (\boldsymbol{k} \boldsymbol{X})+B(\boldsymbol{k}) \sin (\boldsymbol{k} \boldsymbol{X})\}$,

$|\boldsymbol{k}|^{2} \neq 0, \quad k_{1,2}=2 \pi l, \quad l=0, \pm 1, \pm 2$.

The amplitudes $A$ and $B$ are Gaussian random numbers with a standard deviation of 1 around the mean value $0 ; q_{\mathrm{o}}$ is the largest wavelength of the perturbations $2 \pi / k_{\max } \cdot l_{\max }$ related to the cut-off wavelength $2 \pi / k_{\max }=1 / 2 q_{\mathrm{o}}$. We choose $\sigma_{\mathrm{o}}=2$ in accord with the normalization of the special model above.

(c)
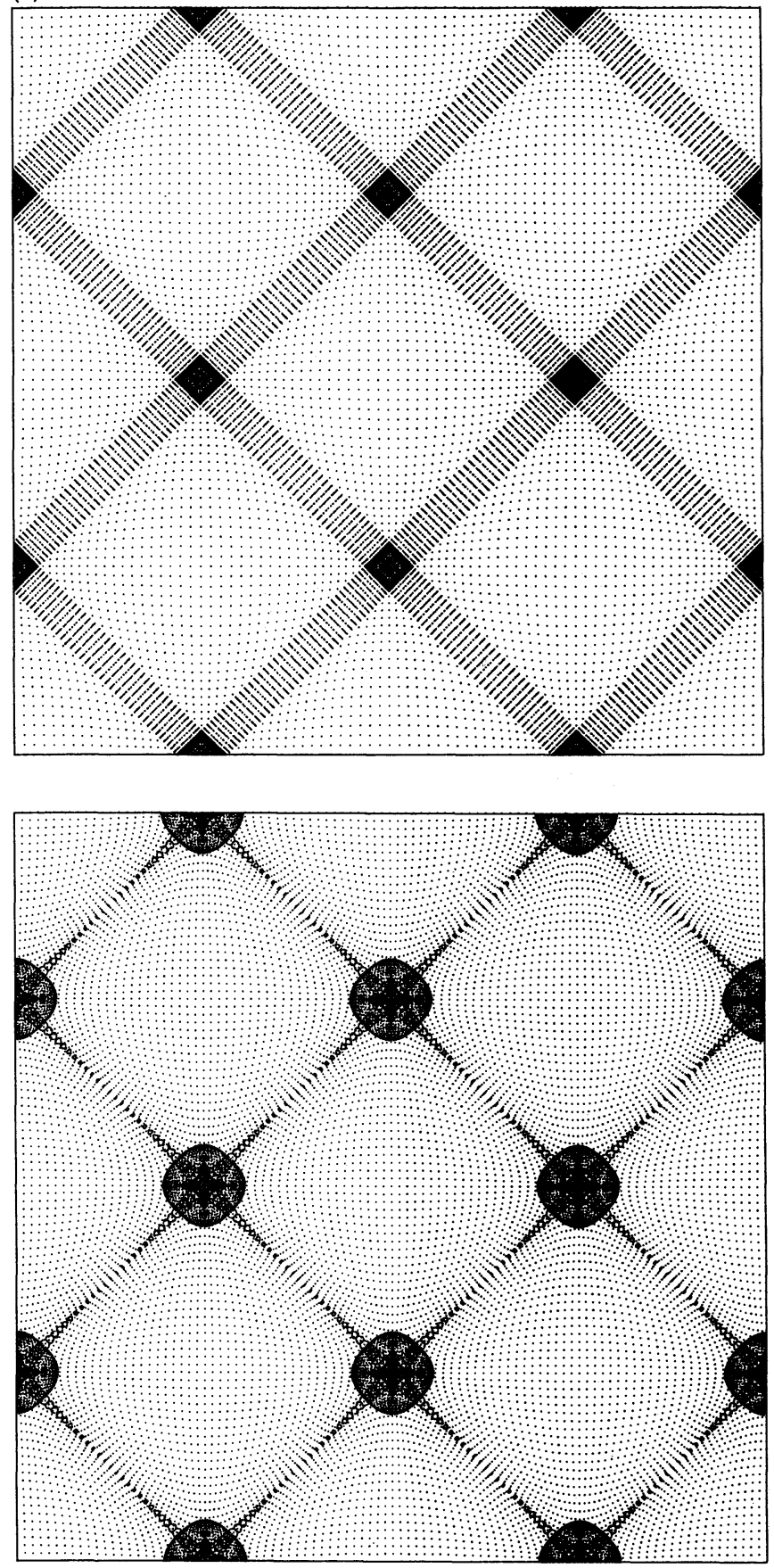

The initial point process on a regular grid is displaced according to mapping (24c), and is shown in Fig. 1 in four consecutive redshift panels for the initial condition (27) in comparison with the corresponding first-order solution (the 'Zel'dovich approximation', in this case). One redshift panel is shown for the corresponding comparison in the generic field (28) in Fig. 2 . In both cases, the closed-form expressions (26) have been used to calculate the initial conditions. Note, however, that for the second illustration artificial vorticity is generated, but this is negligible for the density field. A detailed comparison of the Lagrangian perturbation solu- (d)
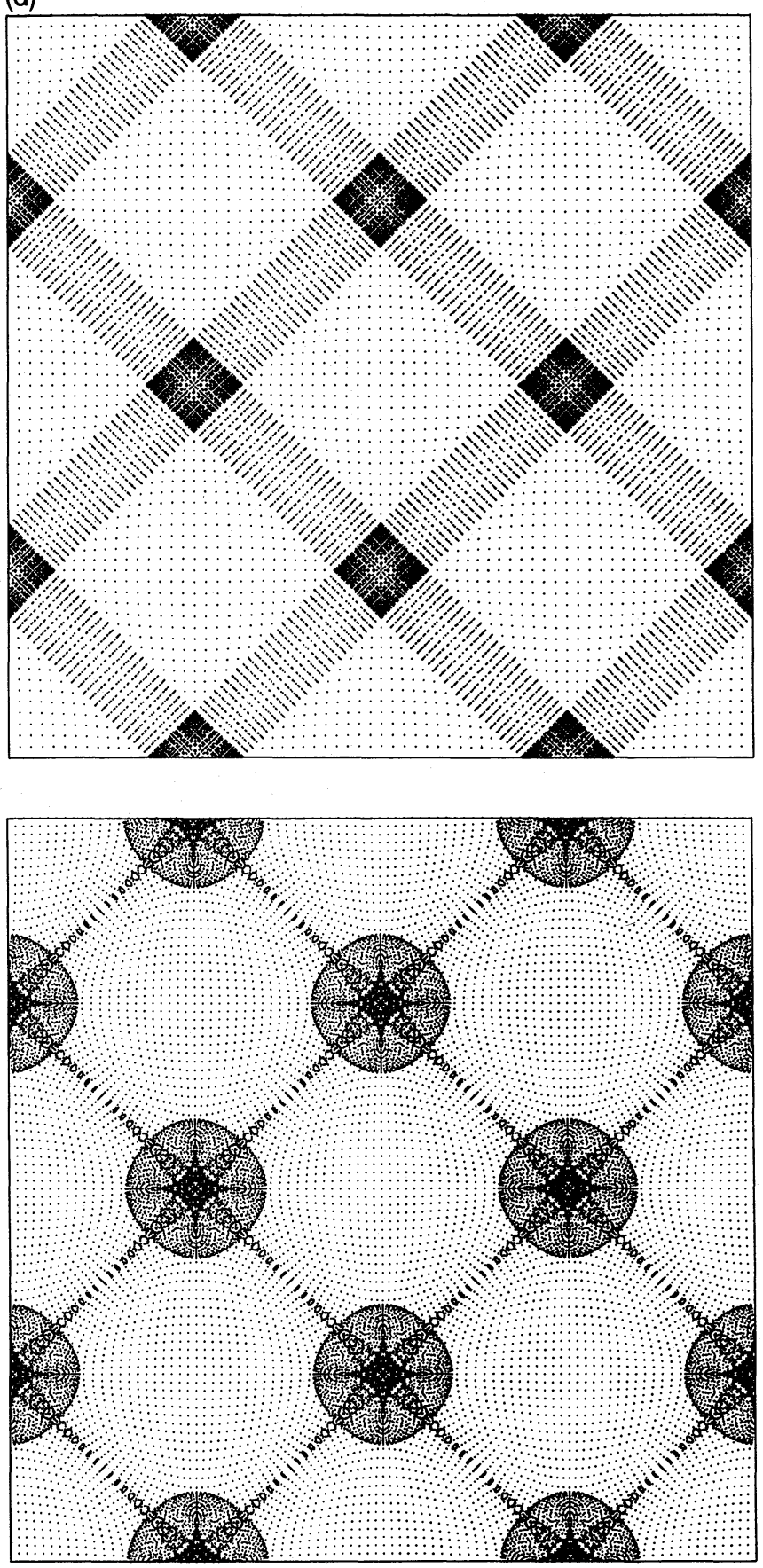

Figure 1 - continued 


\section{T. Buchert and J. Ehlers}

tions with a numerical simulation is postponed until a forthcoming paper.

\subsection{Discussion}

Looking at Figs 1 and 2 we find that the overall picture of the large-scale density field is principally unchanged, but that the thickening of sheet-like structures has not progressed so far

SAMPLE $1 / 1, N G=40000$
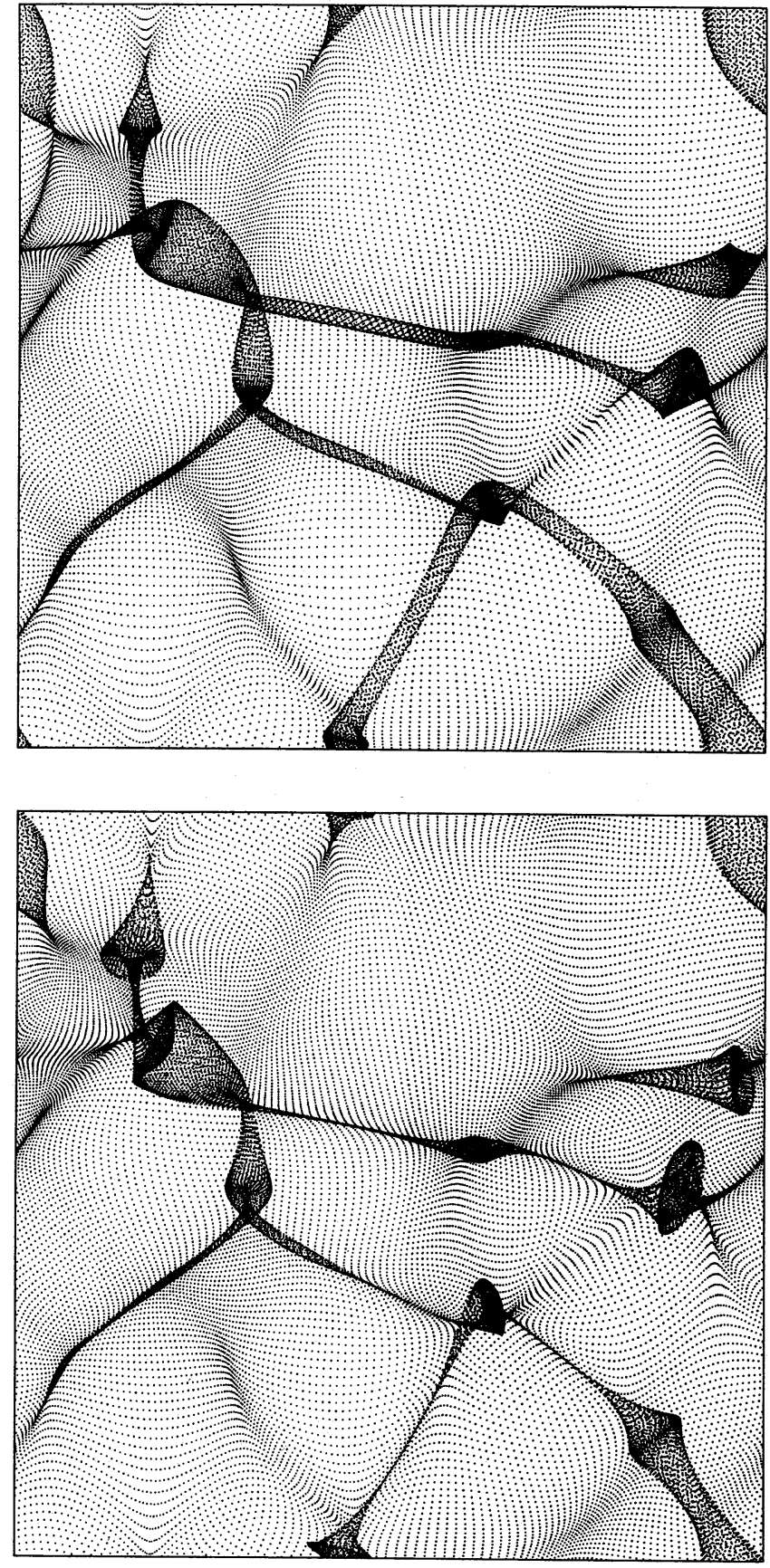

Figure 2. Similar to Fig. 1, but for a generic initial fluctuation field. (Upper and lower plots are as in Fig. 1.) The same effects as described in the caption to Fig. 1 can be seen. The evolutionary stage is $z=0.5$ for the same fluctuation amplitude as in Fig. 1 . in the second-order solution (compare the numerical simulation by Melott \& Shandarin 1989). This compensates for a shortcoming of the first-order solutions (or Zel'dovich's approach, respectively), for which the 'adhesion model' (Gurbatov, Saichev \& Shandarin 1989) was invoked to compensate. On the other hand, the clusters appear rounder and more compact, this being especially visible in Figs 1 and 3.

Another property of the solutions concerns the collapse time of first objects. The collapse occurs earlier in the
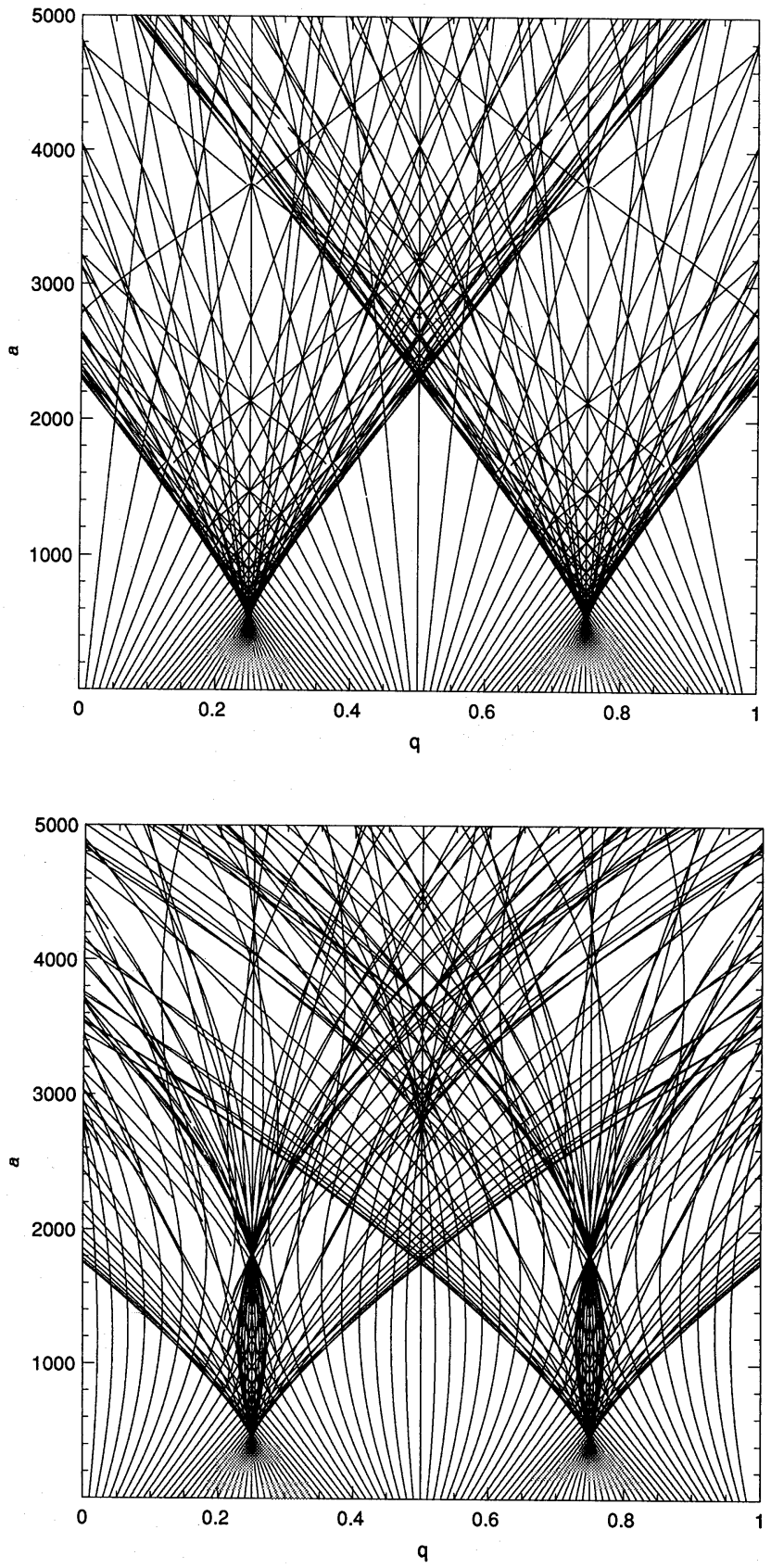

Figure 3. A family of trajectories corresponding to the model presented in Fig. 1 is shown for the first-order (upper panel) and second-order (lower panel) approximations. The trajectories end in the Eulerian space-time section $(y=0.5, t)$ centred at a cluster. These plots illustrate that the three-stream system that develops after the first shell-crossing performs a self-oscillation due to the action of self-gravity. 
second-order theory, an effect that is expected from numerical simulations (e.g. Evrard 1990). Note that the first-order solutions are exact for the case of maximally anisotropic motions (Buchert 1989), which is a bad approximation for the first collapsing objects, which form as a result of nearly spherically symmetric infall [see e.g. Blanchard, Buchert \& Klaffl (1993) for a detailed discussion of the time evolution of first collapsing objects in the 'Zel'dovich approximation' and the spherical top-hat model]. To prepare initial condi- tions for numerical simulations, the second-order approximation (in the form 24c) is therefore more appropriate than the first-order (Zel'dovich) approximation.

Since the second-order solutions not only accelerate the first shell-crossings, but also describe the internal structure of pancakes that results from a second shell-crossing, which occurs after the first one (see Figs 3 and 4), we can expect that higher order Lagrangian perturbations will produce multiple stream-crossings as observed in numerical simula-
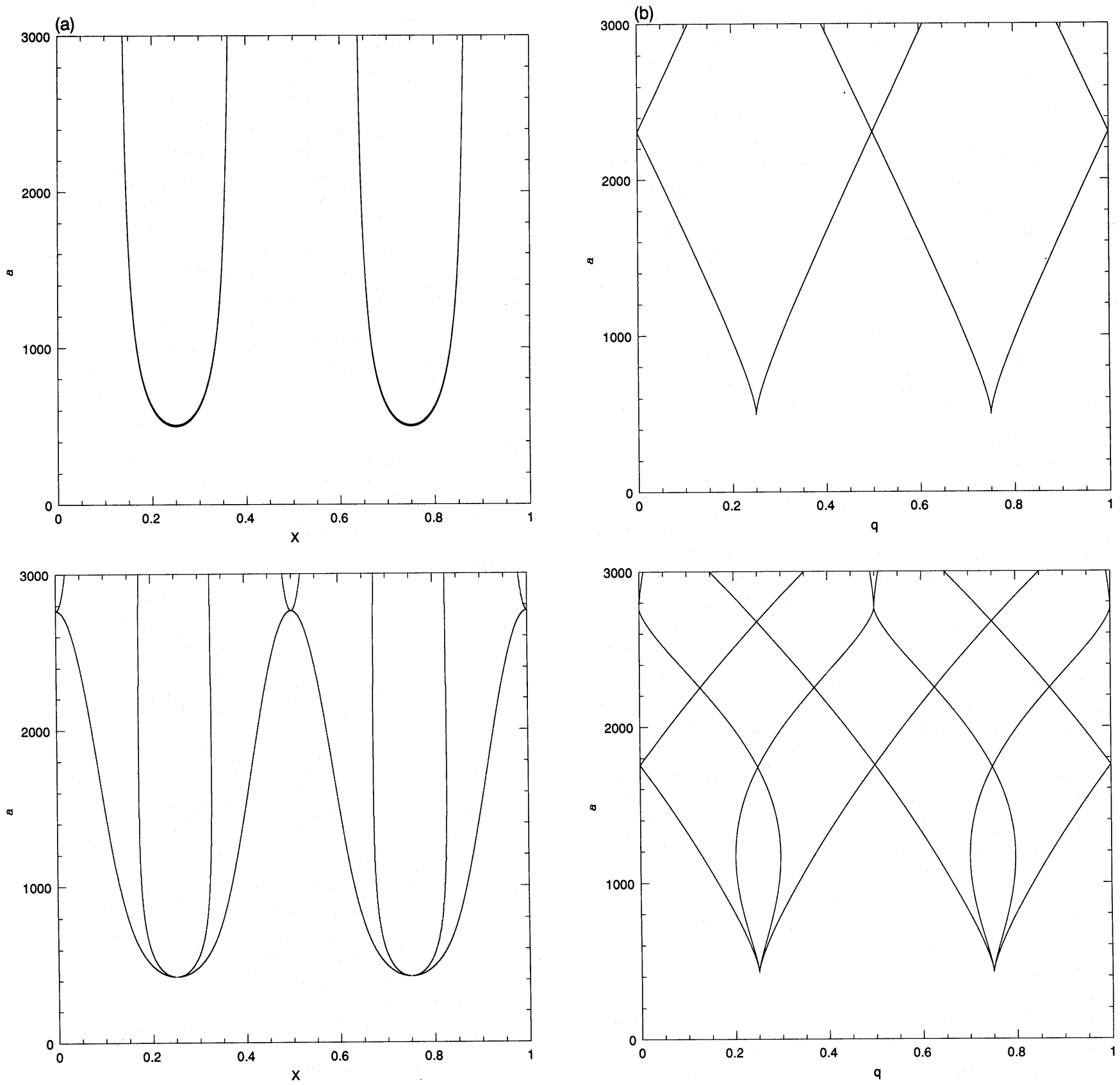

Figure 4. (a) The set of critical points on a slice through the Lagrangian manifold at $Y=0$ for different times (the 'bifurcation diagram') is shown for the first-order (upper panel) and second-order (lower panel) approximations. At the critical points the Jacobian of the transformation from Lagrangian to Eulerian space vanishes. (b) The image of the projection of the critical set in Eulerian space (the 'caustic') shows that the second-order approximation implies a second shell-crossing inside the pancakes [upper and lower panels as in (a)]. The study suggests that higher order Lagrangian perturbations add higher moments of self-oscillations to the multiple-stream system, which in turn can be used to estimate the critical times of breakdown of a solution at a given order. 
tions of the Euler-Poisson system (e.g. Doroshkevich et al. 1980) or the Vlasov-Poisson system (Shukurov 1982; Melott 1983). The comparison of simulations for 'dust' matter with a Vlasov-type description of collisionless matter also shows that solutions of the Euler-Poisson system provide a good approximation for a collisionless medium even after several shell-crossings. The difference is reflected in the finiteness of the density peaks in a Vlasov-type description (due to velocity dispersion acting on smaller scales) compared to the singularities showing up in a pure 'dust' description. It is known that, for regular (i.e. non-'dust') initial conditions, the Vlasov-Poisson system does not develop singularities (Pfaffelmoser 1992). We finally note that the singularities occur in the transformation from Lagrangian to Eulerian space. Since the Lagrangian representation (which relies only on the flow field as the only dynamical variable) does not break down, we can follow the trajectories into regions of multistream flow. Only at Eulerian images of critical points on the Lagrangian manifold is the density field not defined. In order to obtain the correct Eulerian density field, one has to add the moduli of the densities of the individual streams in Eulerian space. This can be achieved numerically by using the ray-tracing method (see Buchert \& Bartelmann 1991), or, in simple cases, by performing the transformation to Eulerian space analytically.

We emphasize that the second-order approximation covers essential effects of the tidal action of the gravitational field, which is completely neglected in the first-order approximation (see Peebles 1987; B92, section 6). The secondorder effects discussed here are related to a long-standing controversy about the importance of tidal fields, non-radial motions and small-scale substructure, or the so-called previrialization effects in gravitational clustering (see Peebles 1990 and references therein). 17 years ago, Peebles \& Groth (1976) expressed their scepticism about the usefulness of the isolated spherical top-hat model as a gauge for the growth rate of inhomogeneities. These authors argued that tides and non-radial motions, absent in the top-hat model but present in the real Universe, will retard the growth of structures. The $\mathrm{N}$-body community responded that the predictions of the top-hat model agree with $N$-body experiments (see e.g. Evrard 1990). Peebles' response to this was that $N$-body simulations do not provide a convincing proof since they may suffer from exactly the kind of problems diagnosed here, namely that tidal actions start to be important quite early, affecting the collapse of protoclusters strongly, while all $\mathrm{N}$ body simulations are initialized with the 'Zel'dovich approximation' at a rather late stage. A solution to this problem could be found by studying the second-order approximation in a quite general form, as derived here. We suggest initializing $N$-body simulations with the second-order approximation and looking for artefacts and transients generated by the first-order approximation in the simulations. Melott (e.g. Melott 1987) emphasized the importance of beginning simulations at a highly linear stage to avoid this problem.

Thus the advantages of the second-order approach over the first-order approximation concern in particular the treatment of tidal forces, the formation of first collapsing objects, the 'adhesive' action on sheet-like structures, and the compactness of clusters. The present investigation suggests that higher order perturbations will subsequently add substructure to the pancakes (defined as three-stream systems of the flow: Arnol'd 1982; Arnol'd, Shandarin \& Zel'dovich 1982) in terms of multiple streams, and will accelerate the collapse of first objects in comparison with the first-order approximation. This extends the validity of the approximation to later times and also to smaller scales. The stage until which the $n$th approximation is valid could roughly be estimated as the time just before the $n$th orbit-crossing sets in. In this regime the Lagrangian perturbation theory is preferred to the 'adhesion model', since the former describes the internal structure of self-gravitating pancakes. Note that the 'adhesion model' is not based on a description of self-gravity, and the momentum in this model is not conserved (the 'viscosity parameter' $v$ is assumed to be spatially constant whereas, in the corresponding equation that preserves momentum, the Navier-Stokes equation with vanishing pressure, the kinematical viscosity $v$ depends on the density, $v=\eta / \rho$ ). This shows that the 'adhesion model' has shortcomings in the case of the simultaneous simulation of objects with different densities, which are overcome by the Lagrangian perturbation theory.

\section{ACKNOWLEDGMENTS}

We would like to thank Günter Götz, Marc B. Wise and Anatoly Klypin for valuable remarks, as well as Peter Schiller for fruitful conversations and the plots of Figs 3 and 4. We are also grateful to the referee for constructive comments and suggestions. TB is supported by DFG (Deutsche Forschungsgemeinschaft).

\section{NOTE ADDED IN PROOF}

A short discussion of the second-order solution for the restriction (24a) is to be found in Buchert (1993a); for the same restriction, the third-order solution is given by Buchert (1993b).

\section{REFERENCES}

Arnol'd V. I., 1982, Trudy Sem. Petrovskogo, 8, 21 (in Russian)

Arnol'd V. I., Shandarin S. F., Zel'dovich Ya.B., 1982, Geophys. Astrophys. Fluid Dyn., 20, 111

Arnol'd V. I., Gusein-Zade S. M., Varchenko A. N., 1985, Singularities of Differentiable Maps, Vol. 1. Birkhäuser, Boston (originally published 1982 by Nauka, Moscow)

Blanchard A., Buchert T., Klaffl R., 1993, A\&A, 267, 1

Bouchet F. R., Juszkiewicz R., Colombi S., Pellat R., 1992, ApJ, 394, L5

Buchert T., 1989, A\&A, 223, 9

Buchert T., 1992, MNRAS, 254, 729

Buchert T., 1993a, A\&A, 267, L51

Buchert T., 1993b, MNRAS, submitted

Buchert T., Bartelmann M., 1991, A\&A, 251, 389

Doroshkevich A. G., Kotok E. V., Novikov I. D., Polyudov A. N., Shandarin S. F., Sigov Yu. S., 1980, MNRAS, 192, 321

Evrard A. E., 1990, ApJ, 363, 349

Friedman A., 1963, Generalized functions and partial differential equations. Prentice Hall, Englewood Cliffs, NJ

Friedman J. L., Schutz B. F., 1978, ApJ, 221, 937

Grinstein B., Wise M. B., 1987, ApJ, 320, 448 [Erratum in ApJ, 337, $579(1989)]$

Gurbatov S. N., Saichev A. I., Shandarin S. F., 1989, MNRAS 236, 385

Lynden-Bell D., Ostriker J. P., 1967, MNRAS, 136, 293

Melott A. L., 1983, ApJ, 264, 59 
Melott A. L., 1987, MNRAS, 282, 1001

Melott A. L., Shandarin S. F., 1989, ApJ, 343, 26

Mo H. J., Buchert T., 1990, A\&A, 234, 5

Moutarde F., Alimi J.-M., Bouchet F. R., Pellat R., Ramani A., 1991, ApJ, 382, 377

Peebles P. J. E., Groth E. J., 1976, A\&A, 53, 131

Peebles P. J. E., 1980, The Large-scale Structure of the Universe. Princeton Univ. Press, Princeton, NJ

Peebles P. J. E., 1987, ApJ, 317, 576

Peebles P. J. E., 1990, ApJ, 365, 27

Pfaffelmoser K., 1992, Differ. Equ., 95, 281

Shukurov A. M., 1982, Astrophys., 17, 263

Zel'dovich Ya. B., 1970, A\&A, 5, 84

Zel'dovich Ya. B., 1973, Astrophys., 6, 164

\section{APPENDIX A}

In this Appendix the constraint equations resulting from the integrability conditions $(5 \mathrm{~d}, \mathrm{e}, \mathrm{f})$ are listed.

We insert the ansatz for longitudinal perturbations

$\boldsymbol{f}=a(t) \boldsymbol{X}+\{\boldsymbol{p}\} ; \quad\{\boldsymbol{p}\}=\varepsilon \nabla_{\mathrm{o}} \psi^{(1)}+\varepsilon^{2} \nabla_{\mathrm{o}} \psi^{(2)}$

into equations $(5 \mathrm{a}, \mathrm{b}, \mathrm{c})$ and obtain, up to the second order,

$$
\begin{aligned}
\omega_{1}= & \varepsilon^{2} a\left[\dot{\psi}_{, 1,3}^{(1)} \psi_{, 1,2}^{(1)}-\dot{\psi}_{, 1,2}^{(1)} \psi_{, 1,3}^{(1)}+\dot{\psi}_{, 2,3}^{(1)} \psi_{, 2,2}^{(1)}-\dot{\psi}_{, 2,2}^{(1)} \psi_{, 2,3}^{(1)}\right. \\
& \left.+\dot{\psi}_{, 3,3}^{(1)} \psi_{, 3,2}^{(1)}-\dot{\psi}_{, 3,2}^{(1)} \psi_{, 3,3}^{(1)}\right] ; \\
\omega_{2}= & \varepsilon^{2} a\left[\dot{\psi}_{, 1,3}^{(1)} \psi_{, 1,1}^{(1)}-\dot{\psi}_{, 1,1}^{(1)} \psi_{, 1,3}^{(1)}+\dot{\psi}_{, 2,3}^{(1)} \psi_{, 2,1}^{(1)}-\dot{\psi}_{, 2,1}^{(1)} \psi_{, 2,3}^{(1)}\right. \\
& \left.+\dot{\psi}_{, 3,3}^{(1)} \psi_{, 3,1}^{(1)}-\dot{\psi}_{, 3,1}^{(1)} \psi_{, 3,3}^{(1)}\right] ; \\
\omega_{3}= & \varepsilon^{2} a\left[\dot{\psi}_{, 1,1}^{(1)} \psi_{, 1,2}^{(1)}-\dot{\psi}_{, 1,2}^{(1)} \psi_{, 1,1}^{(1)}+\dot{\psi}_{, 2,1}^{(1)} \psi_{, 2,2}^{(1)}-\dot{\psi}_{, 2,2}^{(1)} \psi_{, 2,1}^{(1)}\right. \\
& \left.+\dot{\psi}_{, 3,1}^{(1)} \psi_{, 3,2}^{(1)}-\dot{\psi}_{, 3,2}^{(1)} \psi_{, 3,1}^{(1)}\right] .
\end{aligned}
$$

We insert the first-order solution in the form

$\nabla_{\mathrm{o}} \psi^{(1)}=z_{1} \nabla_{\mathrm{o}} \mathscr{S}^{(1)} t_{\mathrm{o}}+z_{2} \nabla_{\mathrm{o}} \phi^{(1)} t_{\mathrm{o}}^{2}$ where $\mathscr{S}(\boldsymbol{X})$ is the peculiar-velocity potential and $\phi(\boldsymbol{X})$ the peculiar-gravity potential (or, in general, any two functions of three independent variables that are related to the initial conditions), and $z_{1}(t)$ and $z_{2}(t)$ are functions of time, which depend on the background solution $a(t)$. We finally obtain

$$
\begin{aligned}
\omega_{1}= & \varepsilon^{2} a\left(\dot{z}_{1} z_{2}-\dot{z}_{2} z_{1}\right)\left[\mathscr{S}_{, 1,3}^{(1)} \phi_{, 1,2}^{(1)}-\mathscr{S}_{, 1,2}^{(1)} \phi_{, 1,3}^{(1)}+\mathscr{S}_{, 2,3}^{(1)} \phi_{, 2,2}^{(1)}\right. \\
& \left.-\mathscr{S}_{, 2,2}^{(1)} \phi_{, 2,3}^{(1)}+\mathscr{S}_{, 3,3}^{(1)} \phi_{, 3,2}^{(1)}-\mathscr{S}_{, 3,2}^{(1)} \phi_{, 3,3}^{(1)}\right] t_{\mathrm{o}}^{3} ; \\
\omega_{2}= & \varepsilon^{2} a\left(\dot{z}_{1} z_{2}-\dot{z}_{2} z_{1}\right)\left[\mathscr{S}_{, 1,3}^{(1)} \phi_{, 1,1}^{(1)}-\mathscr{S}_{, 1,1}^{(1)} \phi_{, 1,3}^{(1)}+\mathscr{S}_{, 2,3}^{(1)} \phi_{, 2,1}^{(1)}\right. \\
& -\mathscr{S}_{, 2,1}^{(1)} \phi_{, 2,3}^{(1)}+\mathscr{S}_{, 3,3}^{(1)} \phi_{, 3,1}^{(1)}-\mathscr{S}_{, 3,1}^{(1)} \phi_{, 3,3}^{(1)} t_{\mathrm{o}}^{3} ; \\
\omega_{3}= & \varepsilon^{2} a\left(\dot{z}_{1} z_{2}-\dot{z}_{2} z_{1}\right)\left[\mathscr{S}_{, 1,1}^{(1)} \phi_{, 1,2}^{(1)}-\mathscr{S}_{, 1,2}^{(1)} \phi_{, 1,1}^{(1)}+\mathscr{S}_{, 2,1}^{(1)} \phi_{, 2,2}^{(1)}\right. \\
& \left.-\mathscr{S}_{, 2,2}^{(1)} \phi_{, 2,1}^{(1)}+\mathscr{S}_{, 3,1}^{(1)} \phi_{, 3,2}^{(1)}-\mathscr{S}_{, 3,2}^{(1)} \phi_{, 3,1}^{(1)}\right] t_{\mathrm{o}}^{3} .
\end{aligned}
$$

The Wronskian $\dot{z}_{1} z_{2}-\dot{z}_{2} z_{1}$ is non-zero for linearly independent solutions. Thus in order to fulfill the constraint equations $(5 \mathrm{~d}, \mathrm{e}, \mathrm{f})$ we have to assure a functional relationship of the form $\nabla_{\mathrm{o}} \phi^{(1)}=\mathscr{F}\left[\nabla_{\mathrm{o}} \mathscr{S}^{(1)}\right]$, or, without loss of generality (see B92),

$\nabla_{\mathrm{o}} \phi=\mathscr{F}\left(\nabla_{\mathrm{o}} \mathscr{S}\right)$

with arbitrary $\mathscr{F}$. There is no restriction for the first-order approximation.

\section{APPENDIX B}

Equation (13c) in B92 is correctly written as follows:

$$
\left(2 \frac{\ddot{a}}{a}-\Lambda\right) q_{p}+\ddot{q}_{p}=\left[\ddot{q}_{p}\left(t_{o}\right)-\ddot{q}_{\ell}\left(t_{o}\right)\right] a^{-2}, \quad \ell=1,2 .
$$

The e-mail address given in the above paper has changed to TOB @ ibma.ipp-garching.mpg.de. 